\title{
Lie-Hamilton systems on the plane: Applications and superposition rules
}

\author{
Alfonso Blasco ${ }^{1}$, Francisco J Herranz ${ }^{1}$, Javier de LuCas $^{2}$ and Cristina Sardón ${ }^{3}$ \\ ${ }^{1}$ Department of Physics, University of Burgos, 09001, Burgos, Spain \\ ${ }^{2}$ Department of Mathematical Methods in Physics, University of Warsaw, Pasteura 5, 02-093, Warszawa, \\ Poland \\ ${ }^{3}$ Department of Fundamental Physics, University of Salamanca, Plaza de la Merced s/n, 37008, Sala- \\ manca, Spain \\ E-mail: ablasco@ubu.es, fjherranz@ubu.es, javier.de.lucas@fuw.edu.pl, \\ cristinasardon@usal.es
}

\begin{abstract}
A Lie-Hamilton system is a nonautonomous system of first-order ordinary differential equations describing the integral curves of a $t$-dependent vector field taking values in a finitedimensional real Lie algebra of Hamiltonian vector fields with respect to a Poisson structure. We provide new algebraic/geometric techniques to easily determine the properties of such Lie algebras on the plane, e.g., their associated Poisson bivectors. We study new and known Lie-Hamilton systems on $\mathbb{R}^{2}$ with physical, biological and mathematical applications. New results cover Cayley-Klein Riccati equations, the here defined planar diffusion Riccati systems, complex Bernoulli differential equations and projective Schrödinger equations. Constants of motion for planar Lie-Hamilton systems are explicitly obtained which, in turn, allow us to derive superposition rules through a coalgebra approach.
\end{abstract}

MSC: 34A26 (primary) 70G45, $70 \mathrm{H} 99$ (secondary)

KEYWORDS: complex Bernoulli differential equation, Casimir element, constant of motion, Lie system, Poisson coalgebra, superposition rule, symplectic geometry, Vessiot-Guldberg Lie algebra 


\section{Introduction}

A Lie system is a nonautonomous system of first-order ordinary differential equations whose general solution can be written as a function, a superposition rule, of a generic family of particular solutions and some constants related to initial conditions [1, 2, 3, 4,. Superposition rules significantly simplify the determination of general solutions for Lie systems as they reduce its derivation to obtaining several particular solutions.

Examples of Lie systems are linear systems of differential equations, Riccati equations and matrix Riccati equations [4, 5, 6. Most nonautonomous systems of first-order ordinary differential equations are not Lie systems [4, 7, 8]. Notwithstanding, Lie systems play a very significant rôle due to their relevant applications and geometric properties, e.g., matrix Riccati equations are ubiquitous in control theory and superposition rules can be understood as a particular type of flat connection [4, 6, 9].

Choose global coordinates $\{x, y\}$ on $\mathbb{R}^{2}$. To study Lie systems on $\mathbb{R}^{2}$, we relate every nonautonomous system

$$
\frac{\mathrm{d} x}{\mathrm{~d} t}=f(t, x, y), \quad \frac{\mathrm{d} y}{\mathrm{~d} t}=g(t, x, y),
$$

where $f, g: \mathbb{R}^{3} \rightarrow \mathbb{R}$ are arbitrary functions, to the $t$-dependent vector field

$$
X: \mathbb{R} \times \mathbb{R}^{2} \ni(t, x, y) \mapsto f(t, x, y) \frac{\partial}{\partial x}+g(t, x, y) \frac{\partial}{\partial y} \in \mathrm{TR}^{2}
$$

and vice versa. This permits us to use $X$ to refer to both (1.1) and (1.2). The Lie-Scheffers Theorem [1, 3] states that $X$ is a Lie system if and only if

$$
X_{t}(x, y):=X(t, x, y)=\sum_{i=1}^{l} b_{i}(t) X_{i}(x, y)
$$

for some $t$-dependent functions $b_{1}(t), \ldots, b_{l}(t)$ and vector fields $X_{1}, \ldots, X_{l}$ on $\mathbb{R}^{2}$ spanning an $l$-dimensional real Lie algebra $V$ of vector fields: a Vessiot-Guldberg Lie algebra of $X$. If $V$ is isomorphic to a Lie algebra $\mathfrak{g}$, we say that $X$ is a $\mathfrak{g}$-Lie system.

As an example of Lie systems, let us consider for the first time the family of nonautonomous complex Bernoulli differential equations [10] of the form

$$
\frac{\mathrm{d} z}{\mathrm{~d} t}=a_{1}(t) z+a_{2}(t) z^{n}, \quad n \notin\{0,1\},
$$

where $z \in \mathbb{C}$ and $a_{1}(t), a_{2}(t)$ are arbitrary complex valued $t$-dependent functions. If $a_{1}(t)$ and $a_{2}(t)$ are polynomial functions of $\mathrm{e}^{\mathrm{i} t}$ and $\mathrm{e}^{-\mathrm{i} t}$, then (1.3) becomes a particular case of the differential equations $\frac{\mathrm{d} z}{\mathrm{~d} t}=P\left(z, \mathrm{e}^{\mathrm{i} t}, \mathrm{e}^{-\mathrm{i} t}\right)$, where $z \in \mathbb{C}$ and $P$ is any polynomial function of their arguments. The number of periodic particular solutions of these latter equations has been studied in [11]. Writing $z=r \mathrm{e}^{\mathrm{i} \theta}, a_{1}(t)=a_{1}^{R}(t)+\mathrm{i} a_{1}^{I}(t)$ and $a_{2}(t)=a_{2}^{R}(t)+\mathrm{i} a_{2}^{I}(t)$ for real $t$-dependent functions $a_{1}^{R}(t), a_{1}^{I}(t), a_{2}^{R}(t), a_{2}^{I}(t)$, system (1.3) becomes

$$
\begin{aligned}
& \frac{\mathrm{d} r}{\mathrm{~d} t}=a_{1}^{R}(t) r+a_{2}^{R}(t) r^{n} \cos [\theta(n-1)]-a_{2}^{I}(t) r^{n} \sin [\theta(n-1)], \\
& \frac{\mathrm{d} \theta}{\mathrm{d} t}=a_{1}^{I}(t)+a_{2}^{R}(t) r^{n-1} \sin [\theta(n-1)]+a_{2}^{I}(t) r^{n-1} \cos [\theta(n-1)],
\end{aligned}
$$


which is related to $X=a_{1}^{R}(t) X_{0}+a_{1}^{I}(t) X_{1}+a_{2}^{R}(t) X_{2}+a_{2}^{I}(t) X_{3}$, where

$$
\begin{aligned}
& X_{0}=r \frac{\partial}{\partial r}, \quad X_{1}=\frac{\partial}{\partial \theta}, \quad X_{2}=r^{n} \cos [\theta(n-1)] \frac{\partial}{\partial r}+r^{n-1} \sin [\theta(n-1)] \frac{\partial}{\partial \theta}, \\
& X_{3}=-r^{n} \sin [\theta(n-1)] \frac{\partial}{\partial r}+r^{n-1} \cos [\theta(n-1)] \frac{\partial}{\partial \theta}
\end{aligned}
$$

span a four-dimensional real Lie algebra, $V^{\mathrm{CB}}$, with commutation relations

$$
\begin{array}{llll}
{\left[X_{0}, X_{1}\right]=0,} & {\left[X_{0}, X_{2}\right]=(n-1) X_{2},} & {\left[X_{0}, X_{3}\right]=(n-1) X_{3},} \\
{\left[X_{1}, X_{2}\right]=(n-1) X_{3},} & {\left[X_{1}, X_{3}\right]=-(n-1) X_{2},} & {\left[X_{2}, X_{3}\right]=0 .}
\end{array}
$$

So, $X$ takes values in the finite-dimensional Lie algebra $V^{\mathrm{CB}}$ and becomes a Lie system.

The Lie-Scheffers Theorem implies that classifying Lie systems on a fixed manifold amounts to determining all non-diffeomorphic finite-dimensional real Lie algebras of vector fields on it [12, 13. In the XIXth century, Lie accomplished the local classification of such Lie algebras on the plane [14, 15]. González-López, Kamran and Olver reviewed Lie's classification using modern geometric techniques [12]. Their (GKO) classification divides finite-dimensional real Lie algebras of planar vector fields into 28 non-diffeomorphic classes, which can be regarded as a local classification of Lie systems on $\mathbb{R}^{2}[12,13]$. For instance, we see from (1.6) that system (1.4) admits a Vessiot-Guldberg Lie algebra $V^{\mathrm{CB}} \simeq \mathbb{R}^{2} \ltimes \mathbb{R}^{2} \simeq\left\langle X_{0}, X_{1}\right\rangle \ltimes\left\langle X_{2}, X_{3}\right\rangle$. According to the GKO classification, all Lie algebras of this type are locally diffeomorphic to the Lie algebra $\mathrm{P}_{4}$ (cf. [12, 13]). That is why we say that (1.4) is a Lie system of class $\mathrm{P}_{4}$.

In this work we focus on studying planar Lie systems possessing a Vessiot-Guldberg Lie algebra of Hamiltonian vector fields with respect to a Poisson structure. It was shown in 13 , lemma 4.1] that this essentially amounts to having a Lie algebra of Hamiltonian vector fields relative to a symplectic structure, a herafter called compatible symplectic structure. Among the 28 classes of the GKO classification, only 12 consist of Lie algebras of Hamiltonian vector fields. Table 1 (see section 2) summarizes the classification of finite-dimensional Lie algebras of Hamiltonian vector fields on $\mathbb{R}^{2}$ given in 13 .

There is not a Lie algebra isomorphic to $V^{\mathrm{CB}}$ in table 1. Consequently, $V^{\mathrm{CB}}$ cannot be a Lie algebra of Hamiltonian vector fields relative to any Poisson structure. This illustrates that not every Lie system admits a Vessiot-Guldberg Lie algebra of Hamiltonian vector fields with respect to a Poisson structure [16, 17]. When a Lie system does, we call it a Lie-Hamilton (LH) system [18]. The interest of LH systems relies on their appearance in physics, mathematics and biology (cf. [13, 18, 19]). Additionally, their associated Poisson structures allow us to study and to derive their constants of motion, superposition rules and Lie symmetries [18, 19].

As an example, consider the complex Bernoulli differential equations (1.4) with $a_{1}^{R}(t)=0$ and the Poisson bivector

$$
\Lambda=X_{2} \wedge X_{3}=r^{2 n-1} \frac{\partial}{\partial r} \wedge \frac{\partial}{\partial \theta}
$$

turning the elements of $V=\left\langle X_{1}, X_{2}, X_{3}\right\rangle$ into Hamiltonian vector fields. Indeed, the Hamiltonian functions for $X_{1}, X_{2}, X_{3}$ read

$$
h_{1}=\frac{1}{(2 n-2) r^{2 n-2}}, \quad h_{2}=\frac{\sin [\theta(n-1)]}{r^{n-1}(n-1)}, \quad h_{3}=\frac{\cos [\theta(n-1)]}{r^{n-1}(n-1)},
$$

correspondingly. These functions along with $h_{0}=1$ fulfill

$$
\left\{h_{1}, h_{2}\right\}_{\Lambda}=-(n-1) h_{3}, \quad\left\{h_{1}, h_{3}\right\}_{\Lambda}=(n-1) h_{2}, \quad\left\{h_{2}, h_{3}\right\}_{\Lambda}=h_{0}, \quad\left\{h_{0}, \cdot\right\}_{\Lambda}=0 .
$$


Hence, system (1.4) with $a_{1}^{R}(t)=0$ is a LH system as it is related to a $t$-dependent vector field taking values in a Vessiot-Guldberg Lie algebra $V$ of Hamiltonian vector fields relative to $\Lambda$. Since $V \simeq \mathbb{R} \ltimes \mathbb{R}^{2} \simeq\left\langle X_{1}\right\rangle \ltimes\left\langle X_{2}, X_{3}\right\rangle, X_{1} \wedge X_{2} \neq 0$ and $\operatorname{ad}_{X_{1}}: X_{i} \in\left\langle X_{2}, X_{3}\right\rangle \mapsto\left[X_{1}, X_{i}\right] \in$ $\left\langle X_{2}, X_{3}\right\rangle$ is not diagonalizable over $\mathbb{R}$, we see in view of table 1 that the Lie algebra $V$ belongs to class $\mathrm{P}_{1}$ and $V \simeq \mathfrak{i s o}(2)$. Meanwhile, the $\mathrm{LH}$ algebra spanned by $h_{1}, h_{2}, h_{3}, h_{0}$ is isomorphic to the centrally extended Euclidean algebra $\overline{\mathfrak{i s o}}(2)$ (see also [13] for further details).

On the other hand, we recall that superposition rules for LH systems can be obtained in an algebraic way by applying a Poisson coalgebra approach [19. In contrast, other methods to derive superposition rules require to integrate a Vessiot-Guldberg Lie algebra, e.g., the group theoretical method [2], or to solve a family of PDEs [9]. Winternitz and coworkers have also derived superposition rules for Lie systems in particular forms [2, 5]. The application of this latter result for general Lie systems requires to map them into the canonical form for which the superposition rule was obtained. The coalgebra procedure makes these transformations unnecessary in many cases.

The structure of the paper is as follows. In section 2 we summarize the local classification of Vessiot-Guldberg Lie algebras as of Hamiltonian vector fields on the plane performed in [13], where the corresponding symplectic structures were derived by solving a system of PDEs. As a first new achievement, we show in section 3 that such symplectic structures can be determined through algebraic and geometric methods. Although our techniques are heavily based upon the Lie algebra structure of Vessiot-Guldberg Lie algebras, they also depend on their geometric properties as Lie algebras of vector fields. In particular, a new method to construct symplectic structures for LH systems on the plane related to non-simple Vessiot-Guldberg Lie algebras is described.

Next we remark that a Vessiot-Guldberg Lie algebra on the plane isomorphic to $\mathfrak{s l}(2)$ can be diffeomorphic to either $\mathrm{P}_{2}, \mathrm{I}_{3}, \mathrm{I}_{4}$ or $\mathrm{I}_{5}$, being $\mathrm{I}_{3}$ the only class which does not consist of Hamiltonian vector fields for any Poisson bivector on $\mathbb{R}^{2}$ (cf. [12]). The problem of determining the existence of diffeomorphisms among $\mathfrak{s l}(2)$-Lie systems on the plane is addressed in section 4. As a second theoretical new result, we provide a new easily verifiable algebraic-geometric criterium allowing one to determine the specific class of a Vessiot-Guldberg Lie algebra on $\mathbb{R}^{2}$ isomorphic to $\mathfrak{s l}(2)$ without finding a change of variables mapping it to a specific class as in [13]. Our new method is based on calculating an easily derivable geometric invariant: a hereafter called Casimir tensor field.

To illustrate the statements of section 4, we retrieve some of the findings in [13] and classify other $\mathfrak{s l}(2)$-LH systems on $\mathbb{R}^{2}$. More specifically, we show in section 5 for the first time that Cayley-Klein Riccati equations [20] comprise the three classes of $\mathfrak{s l}(2)$-LH systems and we study in section 6 their relationships via diffeomorphisms to coupled Riccati equations [21], MilnePinney equations [22], second-order Kummer-Schwarz equations [23] and planar diffusion Riccati systems [24, 25].

In section 7 , we demonstrate that systems described by $t$-dependent quadratic Hamiltonians, e.g. $t$-dependent damped harmonic oscillators or particles under certain electric fields [26, 27], and the second-order Riccati equations in Hamiltonian form [28, 29] can be analyzed through LH systems of class $\mathrm{P}_{5}$ and they become equivalent up to a local diffeomorphism. Such systems admit a $t$-dependent Hamiltonian function taking values in a Lie algebra of Hamiltonian functions isomorphic to the referred to as two-photon algebra [30].

Certain projective Schrödinger equations are studied in section 8 where we prove that they belong to the class $\mathrm{P}_{3}$, that is, they are $\mathfrak{s o}(3)-\mathrm{LH}$ systems. This reveals the interest of $\mathrm{LH}$ systems in geometric quantum mechanics. 
Additionally, we analyze $\mathfrak{h}_{2}$-LH systems in section 9 , where $\mathfrak{h}_{2}$ stands for the two-dimensional Heisenberg Lie algebra 13 which arises within the class $\mathrm{I}_{14 A}$ for $r=1$. As new results we prove that complex Bernoulli differential equations (1.3) with $t$-dependent real coefficients belong to this class and, consequently, we establish their equivalence with generalized Buchdahl equations [31, 32, 33], appearing in Relativity, and $t$-dependent Lotka-Volterra systems [34, 35, occurring in biology.

In section 10 we obtain several superposition rules for LH systems by following the Poisson coalgebra approach [19]. With this aim, we, firstly, obtain $t$-independent constants of motion for LH-systems through Casimir invariants (cf. table 2). And, secondly, we use them in order to construct superposition rules for $\mathrm{LH}$ systems of classes $\mathrm{P}_{1}, \mathrm{P}_{5}$ and $\mathrm{I}_{14 A}^{r=1}$ that were no considered in [19].

Finally, a summary of all the specific LH systems considered throughout the paper (cf. table 3) as well as some open problems are addressed in section 11.

\section{Local classification of LH systems on the plane}

In general, we hereafter assume all structures to be smooth and globally defined. We also consider points where each Poisson bivector $\Lambda$ has locally constant rank. This simplifies the presentation and highlights our main results.

A generic point of a Lie algebra $V$ of vector fields is a point around which the vector fields of $V$ span a regular distribution. We write $\operatorname{dom} V$ for the set of generic points of $V$. Every Lie algebra of planar vector fields is locally diffeomorphic around generic points to one of the 28 classes of vector fields of the GKO classification, which covers two subclasses called primitive (8 cases $\mathrm{P}_{x}$ ) and imprimitive (20 cases $\mathrm{I}_{x}$ ) ones [12].

To determine which of the 28 classes can be considered as Vessiot-Guldberg Lie algebras of Hamiltonian vector fields, a symplectic form

$$
\omega=f(x, y) \mathrm{d} x \wedge \mathrm{d} y
$$

must be found so that each element $X_{i}$ of a basis $\left\{X_{1}, \ldots, X_{l}\right\}$ of the Vessiot-Guldberg Lie algebra under study becomes Hamiltonian (see [13] for details). In such a case, we say that $\omega$ is compatible with the Vessiot-Guldberg Lie algebra. Note that each $X_{i}$ is a Hamiltonian vector field with respect to $\omega$ whenever the Lie derivative of $\omega$ relative to $X_{i}$ vanishes, that is, $\mathcal{L}_{X_{i}} \omega=0$. If $\omega$ exists, then all $X_{i}$ become Hamiltonian vector fields and their corresponding Hamiltonian functions $h_{i}$ are obtained by using the relation $\iota_{X_{i}} \omega=\mathrm{d} h_{i}$. The symplectic form $\omega$ induces a Poisson bracket on $C^{\infty}\left(\mathbb{R}^{2}\right)$ of the form

$$
\{\cdot, \cdot\}_{\omega}: C^{\infty}\left(\mathbb{R}^{2}\right) \times C^{\infty}\left(\mathbb{R}^{2}\right) \ni(f, g) \mapsto X_{g} f \in C^{\infty}\left(\mathbb{R}^{2}\right)
$$

with $X_{g}$ being the Hamiltonian vector field associated to the function $g$. In this way, the functions $h_{1}, \ldots, h_{l}$ and their successive Lie brackets with respect to (2.1) span a finite-dimensional Lie algebra of functions that we call a $L H$ algebra of $V$.

It has been recently proven that the $8+20$ classes of the GKO classification lead to $4+8$ classes of finite-dimensional Lie algebras of Hamiltonian vector fields [13. The final result is summarized in table 1, where we detail the Lie algebra $\mathfrak{g}$ isomorphic to the Vessiot-Guldberg Lie algebra spanned by the vector fields $X_{i}$, an associated symplectic form $\omega$ and the corresponding Hamiltonian functions $h_{i}$. 
Table 1: The classification of the 12 finite-dimensional real Lie algebras of Hamiltonian vector fields on $\mathbb{R}^{2}$. Note that $\mathfrak{g}=\mathfrak{g}_{1} \ltimes \mathfrak{g}_{2}$ means that $\mathfrak{g}$ is the direct sum (as linear subspaces) of $\mathfrak{g}_{1}$ and $\mathfrak{g}_{2}$, with $\mathfrak{g}_{2}$ being an ideal of $\mathfrak{g}$. For $\mathrm{I}_{12}, \mathrm{I}_{14 A}$ and $\mathrm{I}_{16}$, we have $j=1, \ldots, r$ while in $\mathrm{I}_{14 B}$ the index $j=2, \ldots, r$ since $\eta_{1}(x) \equiv 1$. In all cases $r \geq 1$. The elements of the basis of the Lie algebra $\mathrm{P}_{3}$ also admit Hamiltonian functions $\bar{h}_{1}=h_{1}+1 / 4, h_{2}, h_{3}$, respectively, spanning a LH algebra isomorphic to $\mathfrak{s o}(3)$.

\begin{tabular}{lllll}
\hline$\#$ & $\mathfrak{g}$ & Basis of vector fields $X_{i}$ & Hamiltonian functions $h_{i}$ & $\omega$ \\
\hline $\mathrm{P}_{1}$ & $\mathfrak{i s o}(2)$ & $\partial_{x}, \partial_{y}, y \partial_{x}-x \partial_{y}$ & $y,-x, \frac{1}{2}\left(x^{2}+y^{2}\right), 1$ & $\mathrm{~d} x \wedge \mathrm{d} y$ \\
$\mathrm{P}_{2}$ & $\mathfrak{s l}(2)$ & $\partial_{x}, x \partial_{x}+y \partial_{y},\left(x^{2}-y^{2}\right) \partial_{x}+2 x y \partial_{y}$ & $-\frac{1}{y},-\frac{x}{y},-\frac{x^{2}+y^{2}}{y}$ & $\frac{\mathrm{d} x \wedge \mathrm{d} y}{y^{2}}$ \\
$\mathrm{P}_{3}$ & $\mathfrak{s o}(3)$ & $y \partial_{x}-x \partial_{y},\left(1+x^{2}-y^{2}\right) \partial_{x}+2 x y \partial_{y}$, & $\frac{-1}{2\left(1+x^{2}+y^{2}\right)}, \frac{y}{1+x^{2}+y^{2}}$, & $\frac{\mathrm{d} x \wedge \mathrm{d} y}{\left(1+x^{2}+y^{2}\right)^{2}}$ \\
& & $-\frac{x}{1+x^{2}+y^{2}}, 1$ & \\
$\mathrm{P}_{5}$ & $\mathfrak{s l}(2) \ltimes \mathbb{R}^{2}$ & $\partial_{x}, \partial_{y}, x \partial_{x}-y \partial_{y}, y \partial_{x}, x \partial_{y}$ & $y,-x, x y, \frac{1}{2} y^{2},-\frac{1}{2} x^{2}, 1$ & $\mathrm{~d} x \wedge \mathrm{d} y$ \\
$\mathrm{I}_{1}$ & $\mathbb{R}$ & $\partial_{x}$ & $\int^{y} f\left(y^{\prime}\right) \mathrm{d} y^{\prime}$ & $f(y) \mathrm{d} x \wedge \mathrm{d} y$ \\
$\mathrm{I}_{4}$ & $\mathfrak{s l}(2)$ & $\partial_{x}+\partial_{y}, x \partial_{x}+y \partial_{y}, x^{2} \partial_{x}+y^{2} \partial_{y}$ & $\frac{1}{x-y}, \frac{x+y}{2(x-y)}, \frac{x y}{x-y}$ & $\frac{\mathrm{d} x \wedge \mathrm{d} y}{(x-y)^{2}}$ \\
$\mathrm{I}_{5}$ & $\mathfrak{s l}(2)$ & $\partial_{x}, x \partial_{x}+\frac{1}{2} y \partial_{y}, x^{2} \partial_{x}+x y \partial_{y}$ & $-\frac{1}{2 y^{2}},-\frac{x}{2 y^{2}},-\frac{x^{2}}{2 y^{2}}$ & $\frac{\mathrm{d} x \wedge \mathrm{d} y}{y^{3}}$ \\
$\mathrm{I}_{8}$ & $\mathfrak{i s o}(1,1)$ & $\partial_{x}, \partial_{y}, x \partial_{x}-y \partial_{y}$, & $y,-x, x y, 1$ & $\mathrm{~d} x \wedge \mathrm{d} y$ \\
$\mathrm{I}_{12}$ & $\mathbb{R}^{r+1}$ & $\partial_{y}, \xi_{1}(x) \partial_{y}, \ldots, \xi_{r}(x) \partial_{y}$ & $-\int y^{x} f\left(x^{\prime}\right) \mathrm{d} x^{\prime},-\int^{x} f\left(x^{\prime}\right) \xi_{j}\left(x^{\prime}\right) \mathrm{d} x^{\prime}$ & $f(x) \mathrm{d} x \wedge \mathrm{d} y$ \\
$\mathrm{I}_{14 A}$ & $\mathbb{R} \ltimes \mathbb{R}^{r}$ & $\partial_{x}, \eta_{1}(x) \partial_{y}, \ldots, \eta_{r}(x) \partial_{y}$ & $y,-\int \eta_{j}\left(x^{\prime}\right) \mathrm{d} x^{\prime}, 1 \notin\left\langle\eta_{j}\right\rangle$ & $\mathrm{d} x \wedge \mathrm{d} y$ \\
$\mathrm{I}_{14 B}$ & $\mathbb{R} \ltimes \mathbb{R}^{r}$ & $\partial_{x}, \partial_{y}, \eta_{2}(x) \partial_{y}, \ldots, \eta_{r}(x) \partial_{y}$ & $y,-x,-\int \eta_{j}\left(x^{\prime}\right) \mathrm{d} x^{\prime}, 1$ & $\mathrm{~d} x \wedge \mathrm{d} y$ \\
$\mathrm{I}_{16}$ & $\mathfrak{h}_{2} \ltimes \mathbb{R}^{r+1}$ & $\partial_{x}, \partial_{y}, x \partial_{x}-y \partial y, x \partial_{y}, \ldots, x^{r} \partial_{y}$ & $y,-x, x y,-\frac{x^{j+1}}{j+1}, 1$ & $\mathrm{~d} x \wedge \mathrm{d} y$ \\
\hline
\end{tabular}

We remark that in some cases the functions $h_{1}, \ldots, h_{l}$ do not span by themselves a Lie algebra of Hamiltonian functions and a central generator $h_{0}=1$ must be added in such a manner that $\left\langle h_{1}, \ldots, h_{l}, h_{0}\right\rangle$ form a central extension of the initial Vessiot-Guldberg Lie algebra. For instance, the case $\mathrm{P}_{1}$ from table 1 corresponds to the two-dimensional Euclidean algebra $\mathfrak{i s o}(2) \simeq\left\langle X_{1}, X_{2}, X_{3}\right\rangle$, but the Hamiltonian functions $h_{1}, h_{2}, h_{3}, h_{0}=1$ span the centrally extended Euclidean algebra $\overline{\mathfrak{i s o}}(2)$ (as in (1.9)). A similar fact arises in classes $\mathrm{P}_{3} \simeq \mathfrak{s o}(3)$, $\mathrm{P}_{5} \simeq \mathfrak{s l}(2) \ltimes \mathbb{R}^{2}, \mathrm{I}_{8} \simeq \mathfrak{i s o}(1,1)$ (the $(1+1)$-dimensional Poincaré algebra), $\mathrm{I}_{14 B} \simeq \mathbb{R} \ltimes \mathbb{R}^{r}$ and $\mathrm{I}_{16} \simeq \mathfrak{h}_{2} \ltimes \mathbb{R}^{r+1}$. Among them, only the family $\mathrm{P}_{3} \simeq \mathfrak{s o}(3)$ is a simple Lie algebra so that $h_{0}=1$ gives rise to a trivial central extension, namely the LH algebra is $\mathfrak{s o}(3) \oplus \mathbb{R}$; otherwise the central extension is a non-trivial one and it cannot be 'removed' (see [13] for details).

In this respect, notice that the appearance of a non-trivial central extension is the difference between the families $\mathrm{I}_{14 B}$ and $\mathrm{I}_{14 A}$. We also recall that the $\mathrm{LH}$ algebra corresponding to the class $\mathrm{P}_{5}$, that is $\overline{\mathfrak{s l}(2) \ltimes \mathbb{R}^{2}}$, is isomorphic to the two-photon Lie algebra $\mathfrak{h}_{6}$ (see [30, 36] and references therein) and, therefore, to the $(1+1)$-dimensional centrally extended Schrödinger Lie algebra [37.

We stress that the Lie algebra $\mathfrak{s l}(2)$ appears three times (classes $\mathrm{P}_{2}, \mathrm{I}_{4}$ and $\mathrm{I}_{5}$ ) which means that there are different LH systems sharing isomorphic Vessiot-Guldberg Lie algebras that are non-diffeomorphic, that is, there exists no diffeomorphism mapping the elements of one into 
the other. In other words, only LH systems belonging to each class can be related through a $t$-independent change of variables. We shall explicitly apply this property throughout the paper. In section 4 we develop new criteria to easily determine to which class is diffeomorphic a Vessiot-Guldberg Lie algebra isomorphic to $\mathfrak{s l}(2)$ on the plane.

\section{Determination of non-simple LH systems}

The standard approach for determining a symplectic form $\omega$ turning the elements of a VessiotGuldberg Lie algebra $V$ into local Hamiltonian vector fields consists in solving the family of PDEs in $\omega$ of the form $\mathcal{L}_{X_{i}} \omega=0$ with $X_{i}$ being any element of $V$ [13. Meanwhile, we here show how we can derive $\omega$ out of the Lie algebra and geometric structure of $V$ for non-simple planar Vessiot-Guldberg Lie algebras not diffeomorphic either to the trivial Lie algebra $\mathrm{I}_{1}$ or to the Abelian one $\mathrm{I}_{12}$.

Given an $m$-dimensional manifold $M$, a multivector field on $M$ is an element of the $C^{\infty}(M)$ module $\mathfrak{X}^{\bullet} M$ of totally antisymmetric contravariant tensor fields on $M$ of any order. Totally $k$-contravariant multivector fields are called $k$-multivector fields and, when $k=2$, bivector fields. We write $\mathfrak{X}^{k} M$ for the $C^{\infty}(M)$-module of $k$-multivector fields, $\mathfrak{X}^{0} M$ stands for $C^{\infty}(M)$ and we fix $\mathfrak{X}^{k} M=\{0\}$ for $k>\operatorname{dim} M$ and $k<0$. The space $\mathfrak{X}^{\bullet} M$ becomes a $\mathbb{Z}$-graded algebra with respect to the decomposition $\mathfrak{X}^{\bullet} M=\bigoplus_{i \in \mathbb{Z}} \mathfrak{X}^{i} M$ when endowed with the $C^{\infty}(M)$-bilinear exterior product $\wedge: \mathfrak{X}^{\bullet} M \times \mathfrak{X}^{\bullet} M \rightarrow \mathfrak{X}^{\bullet} M$ satisfying

$$
(P \wedge Q)\left(\theta_{1}, \ldots, \theta_{p+q}\right):=\sum_{\sigma \in S_{p+q}}(-1)^{\operatorname{sign}(\sigma)} P\left(\theta_{\sigma(1)}, \ldots, \theta_{\sigma(p)}\right) Q\left(\theta_{\sigma(p+1)}, \ldots, \theta_{\sigma(p+q)}\right),
$$

with $S_{p+q}$ being the permutation group of $p+q$ elements, $P \in \mathfrak{X}^{p} M, Q \in \mathfrak{X}^{q} M$, and $\theta_{1}, \ldots, \theta_{p+q}$ being arbitrary one-forms on $M[16,17]$.

The natural Lie algebra structure on the space $\mathfrak{X}^{1} M$ of vector fields on $M$ can be extended to an $\mathbb{R}$-bilinear operation $[\cdot, \cdot]_{\mathrm{SN}}: \mathfrak{X}^{\bullet} M \times \mathfrak{X}^{\bullet} M \rightarrow \mathfrak{X}^{\bullet} M$ by requiring $\mathfrak{X}^{\bullet} M$ to become a graded Lie algebra relative to the decomposition $\mathfrak{X}^{\bullet} M=\bigoplus_{i \in \mathbb{Z}} \mathfrak{X}^{i} M$ and considering each element of $\mathfrak{X}^{i} M$ to have degree $i-1$. The resulting $\mathbb{R}$-bilinear operation is called the Schouten-Nijenhuis bracket [16]. A bivector field $\Lambda$ satisfying that $[\Lambda, \Lambda]_{\mathrm{SN}}=0$ is called a Poisson bivector.

Consider a Lie algebra $\mathfrak{g}$. Let $(T(\mathfrak{g}), \otimes)$ be the tensorial algebra relative to the linear space $\mathfrak{g}$ and let $\mathcal{R}$ be the ideal of $T(\mathfrak{g})$ generated by the elements $\left[v_{1}, v_{2}\right]-\left(v_{1} \otimes v_{2}-v_{2} \otimes v_{1}\right)$ with $v_{1}, v_{2} \in \mathfrak{g}$. We call $U(\mathfrak{g}):=T(\mathfrak{g}) / \mathcal{R}$ a universal enveloping Lie algebra associated to $\mathfrak{g}$. Observe that $\mathfrak{g}$ can naturally be considered as a subspace of $U(\mathfrak{g})$. Since $\mathcal{R}$ is an ideal, the tensorial product of $(T(\mathfrak{g}), \otimes)$ gives rise to an $\mathbb{R}$-bilinear product $\widetilde{\otimes}: U(\mathfrak{g}) \times U(\mathfrak{g}) \rightarrow U(\mathfrak{g})$ turning $U(\mathfrak{g})$ into an $\mathbb{R}$-algebra $(U(\mathfrak{g}), \widetilde{\otimes})$. The Lie algebra structure of $\mathfrak{g}$ can be extended to $U(\mathfrak{g})$ by requiring the extension to become a derivation on each entry with respect to the product in $(U(\mathfrak{g}), \widetilde{\otimes})$. This makes $U(\mathfrak{g})$ into a Lie algebra $\left(U(\mathfrak{g}),[\cdot, \cdot]_{U(\mathfrak{g})}\right)$ [38]. A Casimir element is an element of $U(\mathfrak{g})$ commuting with every element of $\mathfrak{g}$, namely an element in the center of $\left(U(\mathfrak{g}),[\cdot, \cdot]_{U(\mathfrak{g})}\right)$.

Repeating the same process as above for $\mathcal{R}$ being the ideal of $T(\mathfrak{g})$ generated by the elements $v_{1} \otimes v_{2}-v_{2} \otimes v_{1}$ we obtain the symmetric algebra $S(\mathfrak{g})$ of $\mathfrak{g}$. The Lie algebra structure of $\mathfrak{g}$ can be extended to $S(\mathfrak{g})$ turning this space into a Poisson algebra. As before, $\mathfrak{g}$ is naturally embedded within $S(\mathfrak{g})$. Given a Lie algebra monomorphism $\rho: \mathfrak{g} \rightarrow \mathfrak{X}^{1} M$ mapping a basis $\left\{v_{1}, \ldots, v_{l}\right\}$ of $\mathfrak{g}$ into the vector fields $X_{1}, \ldots, X_{l}$, respectively, the linear morphism $\lambda: S(\mathfrak{g}) \rightarrow U(\mathfrak{g})$ mapping $\lambda\left(v_{i_{1}} \cdot \ldots \cdot v_{i_{l}}\right)=1 / l ! \sum_{\sigma \in S_{l}} X_{\sigma\left(i_{1}\right)} \widetilde{\otimes} \ldots \widetilde{\otimes} X_{\sigma\left(i_{l}\right)}$ is a linear isomorphism [19, 39]. This morphism, the symmetryzer morphism, allows us to interpret the elements of $U(\mathfrak{g})$ as symmetric tensorial 
elements of $T(\mathfrak{g})$. Moreover, the above mentioned linear morphism maps the center of $S(\mathfrak{g})$ into the center of $U(\mathfrak{g})$. Hence, if an element of $\mathfrak{g}$ commutes with the whole $S(\mathfrak{g})$ according to the Lie algebra structure of $S(\mathfrak{g})$, then it also commutes with the whole $U(\mathfrak{g})$ respect to its corresponding Lie algebra structure.

Let $V$ be a Vessiot-Guldberg Lie algebra of vector fields on $M$. We define the linear subspace $V \wedge V:=\{X \wedge Y \mid X, Y \in V\} \subset \mathfrak{X}^{2} M$. Since $\mathcal{L}_{X}\left(T_{1} \otimes T_{2}\right)=\mathcal{L}_{X} T_{1} \otimes T_{2}+T_{1} \otimes \mathcal{L}_{X} T_{2}$ for every $X \in V, T_{1}, T_{2} \in \mathfrak{X}^{\bullet} M$, then the Lie derivative is a derivation relative to the exterior product of multivector fields and we can define a Lie algebra representation $\varphi_{V}: X \in V \mapsto L_{X} \in$ $\operatorname{End}(V \wedge V)$ for $L_{X}: \Lambda \in V \wedge V \mapsto \mathcal{L}_{X} \Lambda \in V \wedge V$. Moreover, due to the graded Lie algebra structure of $\mathfrak{X}^{\bullet} M$, the Schouten-Nijenhuis bracket of two bivector fields is a 3 -vector field. Since 3 -vector fields on $\mathbb{R}^{2}$ vanish everywhere, then the Schouten-Nijenhuis bracket of two bivector fields on $\mathbb{R}^{2}$ is zero and all elements of $V \wedge V$ are Poisson bivectors.

Proposition 3.1. Let $V$ be a Vessiot-Guldberg Lie algebra of planar vector fields. The vector fields of $V$ are Hamiltonian with respect to a bivector field $\Lambda \in V \wedge V \backslash\{0\}$ if and only if $V$ admits a one-dimensional trivial Lie algebra representation within $V \wedge V$.

Proof. If $V$ is a Vessiot-Guldberg Lie algebra of Hamiltonian planar vector fields with respect to a bivector field $\Lambda \in V \wedge V \backslash\{0\}$, then $\mathcal{L}_{X} \Lambda=0$ for every $X \in V$ and $\Lambda$ spans a one-dimensional trivial Lie algebra representation of $V$. Conversely, if $V$ acts trivially and irreducibly via $\varphi_{V}$ on a one-dimensional vector subspace $W \subset V \wedge V$, then the Lie derivatives of every $\Lambda \in W \backslash\{0\}$ with respect to the elements of $V$ vanish. As $V$ consists of planar vector fields by assumption and $\Lambda$ is a non-zero bivector field, then the vector fields of $V$ are Hamiltonian relative to $\Lambda$.

Note 3.2. If $\Lambda$ is a zero planar bivector field, then $\mathcal{L}_{X} \Lambda=0$ for every vector field $X \neq 0$ and $\Lambda$ is a Poisson bivector. Nevertheless, the only Hamiltonian vector fields for a zero Poisson bivector are the zero vector fields. So, $X$ is not a Hamiltonian vector field relative to $\Lambda$. That is why $\mathcal{L}_{X} \Lambda=0$ implies that $X$ is Hamiltonian provided $\Lambda \neq 0$.

Note 3.3. The existence of trivial one-dimensional representations of $V$ within $V \wedge V$ depends on the Lie algebra structure of $V$ and also on its geometric structure as a Lie algebra of vector fields. Indeed, isomorphic Vessiot-Guldberg Lie algebras may admit a different number of trivial representations in $V \wedge V$. For instance, $V:=\left\langle\partial_{x}, \partial_{y}\right\rangle \simeq \mathbb{R}^{2}$ gives rise to a unique one-dimensional representation $\left\langle\partial_{x} \wedge \partial_{y}\right\rangle=V \wedge V$, while $V:=\left\langle\partial_{x}, y \partial_{x}\right\rangle \simeq \mathbb{R}^{2}$ does not give rise to any such a representation because $V \wedge V=\{0\}$.

Example 3.4. Consider the Lie algebra $V:=\mathrm{I}_{14 B}$. In view of table 1, the Lie derivatives of $\Lambda:=\partial_{x} \wedge \partial_{y} \in V \wedge V$ with respect to every element of $\mathrm{I}_{14 B}$ vanish. Indeed,

$$
\left[\partial_{x}, \partial_{x} \wedge \partial_{y}\right]_{\mathrm{SN}}=\left[\partial_{y}, \partial_{x} \wedge \partial_{y}\right]_{\mathrm{SN}}=0, \quad\left[\eta_{j}(x) \partial_{y}, \partial_{x} \wedge \partial_{y}\right]_{\mathrm{SN}}=-\frac{\partial \eta_{j}}{\partial x} \partial_{y} \wedge \partial_{y}=0, \quad j=2, \ldots, r .
$$

This turns $W=\left\langle\partial_{x} \wedge \partial_{y}\right\rangle$ into a trivial one-dimensional representation of $V$. Proposition 3.1 entails that $V$ consists of Hamiltonian vector fields relative to $\Lambda$. This retrieves the result of table 1, where we find the symplectic structure, $\mathrm{d} x \wedge \mathrm{d} y$, induced by $\Lambda$. Moreover, every Lie algebra $\mathrm{I}_{14 A}$ can be extended to a Lie algebra $\mathrm{I}_{14 B}$ by adding the vector field $\partial_{y}$. Applying the above procedure, we obtain the same canonical symplectic structure turning the elements of $V$ into Hamiltonian vector fields.

Example 3.5. Let us turn to the Lie algebra $V:=I_{16}$. From table 1, we have

$$
\begin{gathered}
{\left[\partial_{x}, \partial_{x} \wedge \partial_{y}\right]_{\mathrm{SN}}=0, \quad\left[x \partial_{x}-y \partial_{y}, \partial_{x} \wedge \partial_{y}\right]_{\mathrm{SN}}=-\partial_{x} \wedge \partial_{y}+\partial_{x} \wedge \partial_{y}=0,} \\
{\left[\partial_{y}, \partial_{x} \wedge \partial_{y}\right]_{\mathrm{SN}}=0, \quad\left[x^{j} \partial_{y}, \partial_{x} \wedge \partial_{y}\right]_{\mathrm{SN}}=-j x^{j-1} \partial_{y} \wedge \partial_{y}=0, \quad j=1, \ldots, r .}
\end{gathered}
$$


Hence, $W=\left\langle\partial_{x} \wedge \partial_{y}\right\rangle \subset V \wedge V$ is a trivial one-dimensional representation of $V$. As a consequence, $\partial_{x} \wedge \partial_{y}$ turns all the elements of $W$ into Hamiltonian vector fields. This is equivalent to the result given in table 1 .

The question now is how to determine one-dimensional trivial representations of $V$ in $V \wedge V$. We next devise a method to obtain them for most of the non-simple Lie algebras of table 1 .

Theorem 3.6. If $V$ is a planar Vessiot-Guldberg Lie algebra admitting a two-dimensional ideal $I$ such that $I \wedge I \neq\{0\}$ and the elements of $V$ act on $I$ by traceless operators, namely the mappings $\vartheta_{X}: Y \in I \mapsto[X, Y] \in I$ are traceless for each $X \in V$, then $V$ becomes a Lie algebra of Hamiltonian vector fields with respect to every element of $I \wedge I \backslash\{0\}$.

Proof. If $I \wedge I \neq\{0\}$, the two-dimensional ideal $I=\left\langle Y_{1}, Y_{2}\right\rangle$ gives rise to a one-dimensional space $I \wedge I$. Since $I$ is an ideal, the Lie brackets of elements $X \in V$ with elements of $I$ belong to $I$. This ensures that the mappings $\vartheta_{X}$ are well defined: the Lie derivative with respect to every element of $V$ of an element of $I \wedge I$ belongs to $I \wedge I$. As $I \wedge I \neq\{0\}$ and $V$ consists of planar vector fields, then $Y_{1} \wedge Y_{2} \neq 0$ and we can define the dual one-forms $\left\{\theta_{1}, \theta_{2}\right\}$ to $\left\{Y_{1}, Y_{2}\right\}$. Moreover, $\theta_{1} \wedge \theta_{2}$ is a volume form on $\mathbb{R}^{2}$ and we have that

$$
\begin{aligned}
\mathcal{L}_{X}\left(Y_{1} \wedge Y_{2}\right) & =\left(\mathcal{L}_{X} Y_{1}\right) \wedge Y_{2}+Y_{1} \wedge\left(\mathcal{L}_{X} Y_{2}\right) \\
& =\left[\left(\theta_{1} \wedge \theta_{2}\right)\left(\vartheta_{X} Y_{1}, Y_{2}\right)+\left(\theta_{1} \wedge \theta_{2}\right)\left(Y_{1}, \vartheta_{X} Y_{2}\right)\right] Y_{1} \wedge Y_{2} \\
& =\operatorname{Tr}\left(\vartheta_{X}\right) Y_{1} \wedge Y_{2} \\
& =0 .
\end{aligned}
$$

Since $\Lambda:=Y_{1} \wedge Y_{2} \neq 0$ is a planar bivector field, then $X$ becomes a Hamiltonian vector field relative to $\Lambda$ (or to any non-zero bivector field of $V \wedge V$ ).

Note 3.7. Observe that the condition $I \wedge I \neq\{0\}$ is unavoidable so as to ensure that $V$ admits a compatible Poisson bivector within $V \wedge V \backslash\{0\}$. For instance, the Lie algebra $\mathrm{I}_{19}$ with $r=1$ of vector fields of the GKO classification (see [12, 13]) takes the form $\mathrm{I}_{19}=\left\langle\partial_{x}, \partial_{y}, x \partial_{y}, 2 x \partial_{x}+\right.$ $\left.y \partial_{y}, x^{2} \partial_{x}+x y \partial_{y}\right\rangle$. Note that $I:=\left\langle\partial_{y}, x \partial_{y}\right\rangle \simeq \mathbb{R}^{2}$ is an ideal of $\mathrm{I}_{19}$ and the elements of $\mathrm{I}_{19}$ act on $I$ as traceless operators. Hence, $\mathrm{I}_{19}$ satisfies all conditions of theorem 3.6 apart from the fact that $I \wedge I=\{0\}$. In view of table 1, this Lie algebra does not admit a compatible symplectic structure. Hence, the lack of condition $I \wedge I \neq\{0\}$ makes theorem 3.6 to be false.

Let us now show how the above statement allows us to determine many of the Poisson bivector structures appearing in the table 1 .

Example 3.8. The Lie algebra $\mathrm{P}_{1}$ admits a two-dimensional ideal $I=\left\langle\partial_{x}, \partial_{y}\right\rangle$ satisfying that $I \wedge I \neq\{0\}$. Moreover, $\partial_{x}, \partial_{y}, y \partial_{x}-x \partial_{y}$ act as traceless operators on $I$. In view of theorem 3.6 , the basis $\partial_{x} \wedge \partial_{y}$ of $I \wedge I$ becomes a Poisson bivector turning $\mathrm{P}_{1}$ into a Lie algebra of Hamiltonian vector fields. Observe that this Poisson bivector gives rise to a symplectic form $\omega=\mathrm{d} x \wedge \mathrm{d} y$, which is the one described in table 1 .

Example 3.9. Let us turn to the Lie algebra $\mathrm{P}_{5}$. We have that $I=\left\langle\partial_{x}, \partial_{y}\right\rangle$ is an ideal of $\mathrm{P}_{5}$ with $I \wedge I \neq 0$ and it is straightforward to prove that all elements of $\mathrm{P}_{5}$ act as traceless linear operators on $I$. Hence, theorem 3.6 ensures that $\mathrm{P}_{5}$ is a Lie algebra of Hamiltonian vector fields relative to the basis $\Lambda:=\partial_{x} \wedge \partial_{y}$ of $I \wedge I$. As in the previous example, the symplectic form associated to $\Lambda$ is the canonical one described in table 1 . 
Example 3.10. The Lie algebra $\mathrm{I}_{8}$ possesses an ideal $I=\left\langle\partial_{x}, \partial_{y}\right\rangle$ with $I \wedge I \neq\{0\}$ and all the elements of $\mathrm{I}_{8}$ act on $I$ as traceless mappings. Hence, theorem 3.6 ensures that $\mathrm{I}_{8}$ is a Lie algebra of Hamiltonian vector fields relative to the basis $\Lambda:=\partial_{x} \wedge \partial_{y}$ of $I \wedge I$.

Example 3.11. Consider the Lie algebra of the class $\mathrm{I}_{14 B}^{r=2}$ given by $V:=\left\langle\partial_{x}, \partial_{y}, x \partial_{y}\right\rangle$. This Lie algebra possesses an ideal $I=\left\langle\partial_{x}, \partial_{y}\right\rangle$ satisfying that $I \wedge I \neq\{0\}$. Moreover, the elements of $V$ act on $I$ as traceless operators. Hence, theorem 3.6 ensures that this Lie algebra again consists of Hamiltonian vector fields relative to $\Lambda:=\partial_{x} \wedge \partial_{y}$.

As a practical application, let us apply theorem 3.6 to the subalgebra $V$ appearing in the study of Bernoulli differential equations (1.4) with $a_{1}^{R}(t)=0$. Observe that $V=\left\langle X_{1}, X_{2}, X_{3}\right\rangle$, where $X_{1}, X_{2}, X_{3}$ are given in (1.5), admits an ideal $I=\left\langle X_{2}, X_{3}\right\rangle$. We also have $X_{2} \wedge X_{3} \neq 0$ and the elements of $V$ act on $I$ as traceless operators. So, $V$ satisfies the conditions given in theorem 3.6 and there exists a non-zero Poisson bivector $\Lambda:=X_{2} \wedge X_{3} \in V \wedge V$ given by (1.7) turning the elements of $V$ into Hamiltonian vector fields. Thus, Bernoulli differential equations (1.4) with $a_{1}^{R}(t)=0$ are LH systems and a compatible symplectic structure can be derived out of $V$.

Note that the Lie algebra structure of Vessiot-Guldberg Lie algebras does not characterize by itself the existence of a compatible symplectic structure. This is evident from the GKO classification [12, 13, where isomorphic Vessiot-Guldberg Lie algebras admit different families of compatible symplectic structures depending on whether they are diffeomorphic or not.

\section{Determination of $\mathfrak{s l}(2)$-Lie systems}

As already commented, $\mathfrak{s l}(2)$-Lie systems on the plane can belong to one of the four nondiffeomorphic classes $\mathrm{P}_{2}, \mathrm{I}_{3}, \mathrm{I}_{4}$ and $\mathrm{I}_{5}$. This is related to the fact that the Lie group actions induced by integrating such Lie algebras are not equivariant under a diffeomorphism on $\mathbb{R}^{2}$.

We now provide a method to determine to which class of the GKO classification belongs a Lie algebra $V$ of planar vector fields isomorphic to $\mathfrak{s l}(2)$. When the vector fields of $V$ additionally generate a distribution of rank one, $V$ must be diffeomorphic to $\mathrm{I}_{3}$ since this is the only Lie algebra, among $\mathrm{I}_{3}, \mathrm{I}_{4}, \mathrm{P}_{2}$ and $\mathrm{I}_{5}$, satisfying this property (cf. [12]). The problem to be solved is therefore to classify $V$ when its vector fields generate a distribution of rank two.

Although in [13] several diffeomorphisms among some $\mathfrak{s l}(2)$-LH systems on the plane, and their corresponding Vessiot-Guldberg Lie algebras, were explicitly determined, we here provide a new easily verifiable criterium to ensure to which specific class a planar Vessiot-Guldberg Lie algebra isomorphic to $\mathfrak{s l}(2)$ is diffeomorphic to. This is done with no need of deriving the specific diffeomorphism.

Lemma 4.1. Let $V$ be a Vessiot-Guldberg Lie algebra isomorphic to $\mathfrak{s l}(2)$. We define $S_{2}(V)$ to be the space of 2-contravariant tensor fields spanned by the linear combinations of the tensor fields in the form $X \otimes Y+Y \otimes X$, with $X, Y \in V$, and we write $S_{2}(\mathfrak{s l}(2))$ for the space of polynomials of second order in $S(\mathfrak{s l}(2))$. Choose basis $\left\{v_{1}, v_{2}, v_{3}\right\}$ and $\left\{X_{1}, X_{2}, X_{3}\right\}$ of $\mathfrak{s l}(2)$ and $V$, respectively, with the same structure constants. This gives rise to an isomorphism $T: \mathfrak{s l}(2) \simeq V$ and a morphism of $\mathfrak{s l}(2)$-modules

$$
\Psi: S_{2}(\mathfrak{s l}(2)) \ni \sum_{1=i \leq j}^{3} c_{i j} v_{i} v_{j} \mapsto \sum_{1=i \leq j}^{3} c_{i j}\left(X_{i} \otimes X_{j}+X_{j} \otimes X_{i}\right) \in S_{2}(V),
$$


i.e., $\Psi$ is linear and $\Psi\left(\{v, P\}_{S(\mathfrak{s l}(2))}\right)=\mathcal{L}_{T(v)} \Psi(P)$ for every $v \in \mathfrak{s l}(2)$ and $P \in S_{2}(\mathfrak{s l}(2))$. If $V$ is diffeomorphic to either $\mathrm{P}_{2}, \mathrm{I}_{4}$ or $\mathrm{I}_{5}$, then $\Psi$ is an isomorphism. If $V$ is diffeomorphic to $\mathrm{I}_{3}$, then $\Psi$ is not an isomorphism.

Proof. It is immediate that $\Psi$ is linear by construction. Let us prove that $\Psi$ is a morphism of $\mathfrak{s l}(2)$-modules. Assuming $\left\{v_{i}, v_{j}\right\}_{S(\mathfrak{s l}(2))}=\sum_{k=1}^{3} c_{i j k} v_{k}$, we obtain $\left[X_{i}, X_{j}\right]=\sum_{k=1}^{3} c_{i j k} X_{k}$ and

$$
\begin{aligned}
\Psi\left(\left\{v_{k}, v_{i} v_{j}\right\}_{S(\mathfrak{s l}(2)}\right) & =\Psi\left(\left\{v_{k}, v_{i}\right\}_{S(\mathfrak{s l}(2))} v_{j}+v_{i}\left\{v_{k}, v_{j}\right\}_{S(\mathfrak{s l}(2))}\right)=\Psi\left(\sum_{l=1}^{3} c_{k i l} v_{l} v_{j}+\sum_{l=1}^{3} v_{i} c_{k j l} v_{l}\right) \\
& =\sum_{l=1}^{3} c_{k i l}\left(X_{l} \otimes X_{j}+X_{j} \otimes X_{l}\right)+\sum_{l=1}^{3} c_{k j l}\left(X_{i} \otimes X_{l}+X_{l} \otimes X_{i}\right) \\
& =\left(\mathcal{L}_{X_{k}} X_{i}\right) \otimes X_{j}+X_{j} \otimes\left(\mathcal{L}_{X_{k}} X_{i}\right)+X_{i} \otimes\left(\mathcal{L}_{X_{k}} X_{j}\right)+\left(\mathcal{L}_{X_{k}} X_{j}\right) \otimes X_{i} \\
& =\mathcal{L}_{X_{k}}\left(X_{i} \otimes X_{j}+X_{j} \otimes X_{i}\right)=\mathcal{L}_{T\left(v_{k}\right)} \Psi\left(v_{i} v_{j}\right), \quad \forall k, i, j=1,2,3 .
\end{aligned}
$$

Using the linearity of $\Psi$ and the bilinearity of the Poisson bracket in $S_{2}(\mathfrak{s l}(2))$, we obtain that $\Psi$ is an $\mathfrak{s l}(2)$-module morphism. If $V$ is either $\mathrm{P}_{2}, \mathrm{I}_{4}$ or $\mathrm{I}_{5}$ is straightforward to prove the linearly independence over $\mathbb{R}$ of $\Psi\left(v_{1}^{2}\right), \Psi\left(v_{2}^{2}\right), \Psi\left(v_{3}^{2}\right), \Psi\left(v_{1} v_{2}\right), \Psi\left(v_{1} v_{3}\right), \Psi\left(v_{2} v_{3}\right)$. Since $\operatorname{dim} S_{2}(\mathfrak{s l}(2))=$ $\operatorname{dim} S_{2}(V)=6$, then $\Psi$ is an isomorphism. This fact does not change under diffeomorphisms and so it applies to any Lie algebra $V$ diffeomorphic to either $\mathrm{P}_{2}, \mathrm{I}_{4}$ and $\mathrm{I}_{5}$.

If $V$ is $\mathrm{I}_{3}=\left\langle X_{1}:=\partial_{x}, X_{2}:=x \partial_{x}, X_{3}:=x^{2} \partial_{x}\right\rangle$, then $S_{2}(V)$ is spanned by $\left\{x^{\alpha} \partial_{x} \otimes \partial_{x}: \alpha=\right.$ $0, \ldots, 4\}$. In particular,

$$
\Psi\left(v_{1} v_{3}-v_{2}^{2}\right)=X_{1} \otimes X_{3}+X_{3} \otimes X_{1}-2 X_{2} \otimes X_{2}=0
$$

and $\Psi$ is not an isomorphism because $\operatorname{dim} \operatorname{ker} \Psi=1$. These facts do not change under diffeomorphisms so they remain true for any $V$ diffeomorphic to $\mathrm{I}_{3}$.

Lemma 4.2. A Vessiot-Guldberg Lie algebra $V$ diffeomorphic to either $\mathrm{P}_{2}, \mathrm{I}_{4}$ or $\mathrm{I}_{5}$ admits an essentially unique, namely up to proportional constant, $R \in S_{2}(V) \backslash\{0\}$ such that $\mathcal{L}_{X} R=0$ for every $X \in V$.

Proof. It is well known that there exists only one quadratic Casimir (up to a proportional constant) in $U(\mathfrak{s l}(2))$. The $\mathfrak{s l}(2)$-module isomorphism $\lambda: S(\mathfrak{s l}(2)) \simeq U(\mathfrak{s l}(2))$ maps the Casimir $\mathcal{C}$ into an element of $S_{2}(\mathfrak{s l}(2))$. Using the $\mathfrak{s l}(2)$-module isomorphism $S_{2}(V) \simeq S_{2}(\mathfrak{s l}(2))$, we obtain that there exists essentially one element of $S_{2}(V) \backslash\{0\}$ whose Lie brackets with the elements of $V$ vanish. Thus, there exists essentially a unique, i.e., up to proportional constant, $R \in S_{2}(V) \backslash\{0\}$ such that $\mathcal{L}_{X} R=0$ for every $X \in V$.

Definition 4.3. Given a finite-dimensional real Lie algebra of vector fields $V$, we call Casimir tensor field of $V$ an element $R \in S_{2}(V)$ such that $\mathcal{L}_{X} R=0$ for every $X \in V$.

Theorem 4.4. Let $V$ be a Vessiot-Guldberg Lie algebra diffeomorphic to either $\mathrm{P}_{2}$, $\mathrm{I}_{4}$ or $\mathrm{I}_{5}$. Let $R$ be a non-zero Casimir tensor field for $V$. Writing $R=\sum_{\alpha, \beta=1}^{2} R^{\alpha \beta} \partial_{\alpha} \otimes \partial_{\beta}$ with $\partial_{1}=\partial_{x}$ and $\partial_{2}=\partial_{y}$, we define

$$
\mathcal{I}(V):=\operatorname{sign}\left(\operatorname{det}\left(R^{\alpha \beta}(x)\right)\right), \quad \forall x \in \operatorname{dom} V,
$$

where $\operatorname{dom} V$ is the set of generic points of $V$. If $\mathcal{I}(V)>0$, then $V$ is locally diffeomorphic to $\mathrm{P}_{2}$; when $\mathcal{I}(V)<0$, then $V$ is locally diffeomorphic to $\mathrm{I}_{4}$; if $\mathcal{I}(V)=0$, then $V$ is locally diffeomorphic to $\mathrm{I}_{5}$. 
Proof. For the Lie algebras of vector fields $\mathrm{P}_{2}, \mathrm{I}_{4}$ and $\mathrm{I}_{5}$ and using their corresponding bases $\left\{X_{1}, X_{2}, X_{3}\right\}$ detailed in table 1, we get that

$$
R=\frac{1}{2}\left(X_{1} \otimes X_{3}+X_{3} \otimes X_{1}\right)-X_{2} \otimes X_{2}
$$

satisfies that $\mathcal{L}_{X_{i}} R=0$ for $i=1,2,3$ and $R \neq 0$. In view of lemma 4.2, this is essentially the only element of $S_{2}(V) \backslash\{0\}$ satisfying this property. It is a non-zero Casimir tensor field. After a straightforward calculation and using that $\operatorname{dom} \mathrm{P}_{2}=\mathbb{R}_{y \neq 0}^{2}, \operatorname{dom} \mathrm{I}_{4}=\mathbb{R}_{x \neq y}^{2}$ and $\operatorname{dom} \mathrm{I}_{5}=\mathbb{R}_{y \neq 0}^{2}$ (see [13]), we obtain

$$
\begin{gathered}
R\left(\mathrm{P}_{2}\right)=-y^{2}\left[\partial_{x} \otimes \partial_{x}+\partial_{y} \otimes \partial_{y}\right] \Rightarrow \mathcal{I}\left(\mathrm{P}_{2}\right)=1, \quad R\left(\mathrm{I}_{5}\right)=-\frac{y^{2}}{4}\left[\partial_{y} \otimes \partial_{y}\right] \Rightarrow \mathcal{I}\left(\mathrm{I}_{5}\right)=0 \\
R\left(\mathrm{I}_{4}\right)=\frac{1}{2}(x-y)^{2}\left[\partial_{x} \otimes \partial_{y}+\partial_{y} \otimes \partial_{x}\right] \Rightarrow \mathcal{I}\left(\mathrm{I}_{4}\right)=-1 .
\end{gathered}
$$

Observe that the value of $\mathcal{I}(V)$ is independent of the point $x \in \operatorname{dom} V$ where we evaluate $\operatorname{det}\left(R_{\alpha \beta}(x)\right)$ for the Lie algebras $\mathrm{P}_{2}, \mathrm{I}_{4}$ and $\mathrm{I}_{5}$. Hence, $\mathcal{I}(V)$ is well defined. Moreover, since $\mathcal{I}(V)$ depends on the $2 \times 2$ matrix of coefficients of $R$, which is unique up to a non-zero multiplicative constant, we have that the value of $\mathcal{I}(V)$ does not depend on the non-null chosen $R$.

Let us now prove that $\mathcal{I}(V)$ is invariant under diffeomorphisms and, therefore, two diffeomorphic Lie algebras have the same $\mathcal{I}(V)$. Given a local diffeomorphism $\phi: \mathbb{R}^{2} \rightarrow \mathbb{R}^{2}$, we can write that $\phi_{*} R=\sum_{\alpha, \beta=1}^{2} \bar{g}^{\alpha \beta} \partial_{\alpha} \otimes \partial_{\beta}=\sum_{\mu, \nu=1}^{2} g^{\mu \nu} A_{\mu}^{\alpha} A_{\nu}^{\beta} \partial_{\alpha} \otimes \partial_{\beta}$, where $A=\left(A_{\sigma}^{\lambda}\right)$ is the Jacobian matrix of the diffeomorphism in the initial and final basis $\left\{\partial_{x}, \partial_{y}\right\}$. In consequence, $\operatorname{det} \bar{g}^{\alpha \beta}=\operatorname{det} A^{2} \operatorname{det} g^{\alpha \beta}$ and $\mathcal{I}(V)$ is invariant under diffeomorphisms.

If $V$ is diffeomorphic to one of the Lie algebras $\mathrm{P}_{2}, \mathrm{I}_{4}$ or $\mathrm{I}_{5}$, then the element $R$ for $V$ is essentially unique and it must be mapped via a diffeomorphism onto an $R \neq 0$ corresponding to $\mathrm{P}_{2}, \mathrm{I}_{4}$ or $\mathrm{I}_{5}$. Since $\mathcal{I}(V)$ is invariant under diffeomorphisms, we obtain that $\mathcal{I}(V)$ is the same as the one for the Lie algebra to which is diffeomorphic to.

In the next two sections we apply the above results in the study of planar $\mathfrak{s l}(2)-\mathrm{LH}$ systems and their equivalence via diffeomorphisms.

\section{Cayley-Klein Riccati equations}

Let us consider the so-called complex Riccati equations, namely

$$
\frac{\mathrm{d} z}{\mathrm{~d} t}=a_{0}(t)+a_{1}(t) z+a_{2}(t) z^{2}, \quad z \in \mathbb{C},
$$

with arbitrary $t$-dependent real coefficients $a_{0}(t), a_{1}(t)$ and $a_{2}(t)$. These equations have several applications from a mathematical and physical point of view [40, 41, 42]. In fact, these can be mapped into a particular type of planar Riccati equation [43, 44] and they also appear in the study of dissipative and irreversible systems [45]. We hereafter propose a generalization of Riccati equations over the so-called split-complex and dual-Study numbers.

Consider the real plane with coordinates $\{u, v\}$ and an 'additional' unit $\iota$ such that $\iota^{2} \in$ $\{-1,+1,0\}$. Next we define $z:=u+\iota v$ for $(u, v) \in \mathbb{R}^{2}$. Assuming that $\iota$ commutes with real numbers, we can write $z^{2}=u^{2}+\iota^{2} v^{2}+2 \iota u v$. In this way, the number $z$ comprises three possibilities [4]: 
- If $\iota^{2}=-1$, we are dealing with the usual complex numbers $\iota:=\mathrm{i}$ and $z \in \mathbb{C}$.

- Setting $\iota^{2}=+1$ we obtain the so-called split-complex numbers $z \in \mathbb{C}^{\prime}$. The additional unit is usually known as the double or Clifford unit.

- Meanwhile, if we assume $\iota^{2}=0$, then $z$ is known as a dual or Study number, $z \in \mathbb{D}$.

With these ingredients we shall call the Cayley-Klein Riccati equation [20] the generalization of the complex Riccati equation (5.1) to $z:=u+\imath v \in\left\{\mathbb{C}, \mathbb{C}^{\prime}, \mathbb{D}\right\}$ which, for real $t$-dependent coefficients $a_{0}(t), a_{1}(t), a_{2}(t)$, gives rise to the system

$$
\frac{\mathrm{d} u}{\mathrm{~d} t}=a_{0}(t)+a_{1}(t) u+a_{2}(t)\left(u^{2}+\iota^{2} v^{2}\right), \quad \frac{\mathrm{d} v}{\mathrm{~d} t}=a_{1}(t) v+a_{2}(t) 2 u v .
$$

Let us prove that (5.2) is a Lie system for every possible value of $\iota$. The system (5.2) is associated with the $t$-dependent vector field given by

$$
X=a_{0}(t) X_{1}+a_{1}(t) X_{2}+a_{2}(t) X_{3}
$$

where

$$
X_{1}=\frac{\partial}{\partial u}, \quad X_{2}=u \frac{\partial}{\partial u}+v \frac{\partial}{\partial v}, \quad X_{3}=\left(u^{2}+\iota^{2} v^{2}\right) \frac{\partial}{\partial u}+2 u v \frac{\partial}{\partial v},
$$

span a Vessiot-Guldberg Lie algebra $V_{\iota} \simeq \mathfrak{s l}(2)$ with commutation relations given by

$$
\left[X_{1}, X_{2}\right]=X_{1}, \quad\left[X_{1}, X_{3}\right]=2 X_{2}, \quad\left[X_{2}, X_{3}\right]=X_{3}
$$

Consequently, $X$ takes values in a finite-dimensional Lie algebra $V_{\iota}$ of vector fields and (5.2) becomes a Lie system. Let us prove that $X$ is also a LH system.

Proposition 5.1. The Cayley-Klein Riccati equation with $t$-dependent real coefficients (5.2) is a LH system for each value of $\iota$. It admits a Vessiot-Guldberg Lie algebra, $V_{\iota}$, which is locally diffeomorphic around points of $\operatorname{dom} V_{\iota}$ to $\mathrm{P}_{2}$ when $\iota^{2}=-1$, to $\mathrm{I}_{4}$ when $\iota^{2}=1$, and to $\mathrm{I}_{5}$ when $\iota^{2}=0$.

Proof. Since the Vessiot-Guldberg Lie algebra $V_{\iota}$ for Cayley-Klein Riccati equations is spanned by the vector fields (5.4) and their commutation relations are given by (5.5), it turns out that a Casimir tensor field for $V_{\iota}$ is given by (4.1). Substituying (5.4) in (4.1), we obtain that

$$
R=\iota^{2} v^{2} \partial_{u} \otimes \partial_{u}-v^{2} \partial_{v} \otimes \partial_{v} \Rightarrow \mathcal{I}\left(V_{\iota}\right)=-\operatorname{sign}\left(\iota^{2}\right)
$$

for points in $\operatorname{dom} V_{\iota}$. The vector fields of (5.4) span a distribution of rank 2, so $V_{\iota}$ must be diffeomorphic to one of the Lie algebras $\mathrm{P}_{2}, \mathrm{I}_{4}, \mathrm{I}_{5}$. From table 1 we obtain that all these Lie algebras consist of Hamiltonian vector fields. Thus, all Cayley-Klein Riccati equations with real $t$-dependent coefficients are LH systems. Finally, we see in view of theorem 4.4 that $V_{\iota}$ is locally diffeomorphic to $\mathrm{P}_{2}$ for $\iota^{2}=-1$, to $\mathrm{I}_{4}$ for $\iota^{2}=1$, and to $\mathrm{I}_{5}$ for $\iota^{2}=0$.

Proposition 5.1 allows us to classify the Vessiot-Guldberg Lie algebra of Cayley-Klein equations according to the value of $\iota^{2}$. Notice that complex Riccati equations (5.1) are just the Cayley-Klein Riccati equations for $\iota^{2}=-1$ and the vector fields (5.4) coincide with the basis of vector fields of $\mathrm{P}_{2}$ given in table 1 provided that $\{x:=u, y:=v\}$. This suggests us to call split-complex Riccati equations and dual-Study Riccati equations the Cayley-Klein equations 
for $\iota^{2}=1$ and $\iota^{2}=0$, respectively. Next, we make use of this result and table 1 to determine the associated symplectic structure for these two latter cases.

Consider the case $\iota^{2}=1$ and define the new variables $\{x, y\}$ given by

$$
x:=u+v, \quad y:=u-v, \quad u=\frac{1}{2}(x+y), \quad v=\frac{1}{2}(x-y) .
$$

In the new coordinate system, the vector fields (5.4) take the form of the basis of $\mathrm{I}_{4}$ given in table 1 such that dom $\mathrm{I}_{4}=\mathbb{R}_{x \neq y}^{2}=\mathbb{R}_{v \neq 0}^{2}$. Writing the compatible symplectic two-form and the associated Hamiltonian functions for the basis of $\mathrm{I}_{4}$ given in table 1 in the variables $\{u, v\}$, we obtain that $X_{1}, X_{2}, X_{3}$ are Hamiltonian vector fields with Hamiltonian functions $h_{1}, h_{2}, h_{3}$ relative to the symplectic form $\omega$ with

$$
\omega=-\frac{\mathrm{d} u \wedge \mathrm{d} v}{2 v^{2}}, \quad h_{1}=\frac{1}{2 v}, \quad h_{2}=\frac{u}{2 v}, \quad h_{3}=\frac{u^{2}-v^{2}}{2 v} .
$$

Assume now $\iota^{2}=0$ and $v>0$. The case $v<0$ can be studied analogously giving a similar result. We define new variables $\{x, y\}$ of the form

$$
x:=u, \quad y:=\sqrt{v}, \quad u=x, \quad v=y^{2} .
$$

By writing the vector fields (5.4) in the new variables, we obtain the basis of vector fields appearing in the Lie algebra $I_{5} \simeq \mathfrak{s l}(2)$ of table 1 with dom $I_{5}=\mathbb{R}_{y \neq 0}^{2}=\mathbb{R}_{v \neq 0}^{2}$. Hence in the variables $\{u, v\}$, we find that

$$
\omega=\frac{\mathrm{d} u \wedge \mathrm{d} v}{2 v^{2}}, \quad h_{1}=-\frac{1}{2 v}, \quad h_{2}=-\frac{u}{2 v}, \quad h_{3}=-\frac{u^{2}}{2 v} .
$$

We remark that, independently of the value of $\iota^{2}$, the Hamiltonian functions $h_{1}, h_{2}, h_{3}$ satisfy $($ see $(5.5))$

$$
\left\{h_{1}, h_{2}\right\}_{\omega}=-h_{1}, \quad\left\{h_{1}, h_{3}\right\}_{\omega}=-2 h_{2}, \quad\left\{h_{2}, h_{3}\right\}_{\omega}=-h_{3} .
$$

Hence, $\left(\left\langle h_{1}, h_{2}, h_{3}\right\rangle,\{\cdot, \cdot\}_{\omega}\right)$ is always a LH algebra for the system (5.2) isomorphic to $\mathfrak{s l}(2)$ and

$$
h=a_{0}(t) h_{1}+a_{1}(t) h_{2}+a_{2}(t) h_{3}
$$

is a $t$-dependent Hamiltonian function associated to the $t$-dependent vector field (5.3) and, therefore, to the system (5.2).

We also stress that the Cayley-Klein system (5.2) for $\iota^{2} \in\{0,1\}$ appears in a method to map diffusion-type equations into a simpler form which can be easily integrated [24, 25].

\section{$6 \quad$ Other planar $\mathfrak{s l}(2)-\mathbf{L H}$ systems}

In this section we present some $\mathfrak{s l}(2)-\mathrm{LH}$ systems of mathematical and physical interest, namely, coupled Riccati, Milne-Pinney (which comprises the Smorodinsky-Winternitz system and the harmonic oscillator, both with a $t$-dependent frequency), second-order Kummer-Schwarz and planar diffusion equations. Furthermore, we also study, according to table 1, the equivalence among them and the Cayley-Klein Riccati equations introduced in the previous section, that is, we establish, by applying the results of section 4, which of all of the above systems are locally diffeomorphic. To keep notation simple, hereafter we say that a second-order differential equation is a LH system when the first-order system obtained from it by adding a new variable $y:=\mathrm{d} x / \mathrm{d} t$, is a LH one. 


\subsection{Coupled Riccati differential equations}

Consider the system of coupled differential Riccati equations [21]

$$
\frac{\mathrm{d} x}{\mathrm{~d} t}=a_{0}(t)+a_{1}(t) x+a_{2}(t) x^{2}, \quad \frac{\mathrm{d} y}{\mathrm{~d} t}=a_{0}(t)+a_{1}(t) y+a_{2}(t) y^{2},
$$

which appears as a particular case of systems of Riccati equations studied in [19, 47]. This system can be expressed as a $t$-dependent vector field (5.3) where

$$
X_{1}=\frac{\partial}{\partial x}+\frac{\partial}{\partial y}, \quad X_{2}=x \frac{\partial}{\partial x}+y \frac{\partial}{\partial y}, \quad X_{3}=x^{2} \frac{\partial}{\partial x}+y^{2} \frac{\partial}{\partial y},
$$

so that these vector fields exactly reproduce those given in table 1 for the class $\mathrm{I}_{4}$ which, in turn, means that this system is locally diffeomorphic to the split-complex Riccati equation, namely (5.2) with $\iota^{2}=1$.

\subsection{Milne-Pinney equations}

The Milne-Pinney equation [22, 48, 49] has the following expression

$$
\frac{\mathrm{d}^{2} x}{\mathrm{~d} t^{2}}=-\omega^{2}(t) x+\frac{c}{x^{3}}
$$

where $\omega(t)$ is any $t$-dependent function and $c$ is a real constant. We remark that this system is, in fact, the one-dimensional Ermakov system as well as the Smorodinsky-Winternitz system [50] with a $t$-dependent frequency. The $c$-term can be understood as a Rosochatius potential (or a centrifugal barrier when $c>0$ ) in its Hamiltonian form [51]. When $c$ vanishes, the system (6.2) reduces to the harmonic oscillator with a $t$-dependent frequency.

Next, by introducing $y:=\mathrm{d} x / \mathrm{d} t$, we rewrite (6.2) as a system of first-order differential equations

$$
\frac{\mathrm{d} x}{\mathrm{~d} t}=y, \quad \frac{\mathrm{d} y}{\mathrm{~d} t}=-\omega^{2}(t) x+\frac{c}{x^{3}},
$$

which has an associated $t$-dependent vector field $X=X_{3}+\omega^{2}(t) X_{1}$, where

$$
X_{1}=-x \frac{\partial}{\partial y}, \quad X_{2}=\frac{1}{2}\left(y \frac{\partial}{\partial y}-x \frac{\partial}{\partial x}\right), \quad X_{3}=y \frac{\partial}{\partial x}+\frac{c}{x^{3}} \frac{\partial}{\partial y},
$$

span a Lie algebra $V^{\mathrm{MP}}$ of vector fields isomorphic to $\mathfrak{s l}(2)$ with commutation relations given by (5.5). It has been proven in [13] that the Milne-Pinney equations (6.3) comprise the three different types of $\mathfrak{s l}(2)$-LH systems according to the value of the constant $c$ as follows.

Proposition 6.1. The system (6.3) is a LH system of class $\mathrm{P}_{2}$ for $c>0 ; \mathrm{I}_{4}$ for $c<0$; and $\mathrm{I}_{5}$ for $c=0$.

Since $V^{\mathrm{MP}}$ spans a distribution of rank two on the plane and it is isomorphic to $\mathfrak{s l}(2)$, it must be diffeomorphic to $\mathrm{P}_{2}, \mathrm{I}_{4}$ or $\mathrm{I}_{5}$. We can therefore recover, straightforwardly, the above proposition as a particular case of theorem 4.4. Since the vector fields (6.4) satisfy the commutation relations (5.5), we obtain that (4.1) reads

$$
R=-\frac{1}{4}\left[x y\left(\partial_{x} \otimes \partial_{y}+\partial_{y} \otimes \partial_{x}\right)+x^{2} \partial_{x} \otimes \partial_{x}+\left(y^{2}+\frac{4 c}{x^{2}}\right) \partial_{y} \otimes \partial_{y}\right]
$$

and $\mathcal{I}\left(V^{\mathrm{MP}}\right)=c$. Using this and theorem 4.4, we retrieve the same result given in previous proposition. Therefore, like the Cayley-Klein Riccati equations (5.2), the Milne-Pinney ones include the three possibilities of Vessiot-Guldberg Lie algebras isomorphic to $\mathfrak{s l}(2)$ of Hamiltonian vector fields. 


\subsection{Second-order Kummer-Schwarz equation}

This is the second-order differential equation given by

$$
\frac{\mathrm{d}^{2} x}{\mathrm{~d} t^{2}}=\frac{3}{2 x}\left(\frac{\mathrm{d} x}{\mathrm{~d} t}\right)^{2}-2 c x^{3}+2 \eta(t) x,
$$

where $c$ is a real constant and $\eta(t)$ is an arbitrary $t$-dependent function. We define $y:=\mathrm{d} x / \mathrm{d} t$ and we obtain a first-order system which has been studied in 23

$$
\frac{\mathrm{d} x}{\mathrm{~d} t}=y, \quad \frac{\mathrm{d} y}{\mathrm{~d} t}=\frac{3}{2} \frac{y^{2}}{x}-2 c x^{3}+2 \eta(t) x .
$$

This system has an associated $t$-dependent vector field $X=X_{3}+\eta(t) X_{1}$, where the vector fields

$$
X_{1}=2 x \frac{\partial}{\partial y}, \quad X_{2}=x \frac{\partial}{\partial x}+2 y \frac{\partial}{\partial y}, \quad X_{3}=y \frac{\partial}{\partial x}+\left(\frac{3}{2} \frac{y^{2}}{x}-2 c x^{3}\right) \frac{\partial}{\partial y}
$$

form a basis of a Lie algebra $V^{\mathrm{KS}}$ isomorphic to $\mathfrak{s l}(2)$ with commutation relations (5.5).

It can be proven that $V^{\mathrm{KS}}$ comprises, once more, the three Vessiot-Guldberg Lie algebras of Hamiltonian vector fields isomorphic to $\mathfrak{s l}(2)$ given in table 1 according to the value of the parameter $c[13$.

Proposition 6.2. The system (6.5) is a LH system of class $\mathrm{P}_{2}$ for $c>0 ; \mathrm{I}_{4}$ for $c<0$; and $\mathrm{I}_{5}$ for $c=0$.

This statement was obtained in 13 by deriving the explicit diffeomorphisms from the Lie algebra spanned by (6.6) to one of the Lie algebras given in table 1 . In any case, we can retrieve these results more easily by using theorem 4.4. Indeed, as the vector fields (6.6) span a distribution of rank two, the theorem 4.4 applies. Moreover, these vector fields satisfy the commutation relations (5.5) and we find that

$R=\frac{1}{2}\left(X_{1} \otimes X_{3}+X_{3} \otimes X_{1}\right)-X_{2} \otimes X_{2}=-x^{2} \partial_{x} \otimes \partial_{x}-x y\left(\partial_{x} \otimes \partial_{y}+\partial_{y} \otimes \partial_{x}\right)-\left(y^{2}+4 c x^{4}\right) \partial_{y} \otimes \partial_{y}$

Hence, $\mathcal{I}\left(V^{\mathrm{KS}}\right)=c$ and, in view of theorem 4.4, we recover the results given in proposition 6.2 ,

\subsection{Planar diffusion Riccati system}

A diffusion equation can be transformed into a simpler PDE by solving a system of seven firstorder ordinary differential equations (see [24] and [25, p. 104] for details). This system can be easily solved by integrating its projection onto $\mathbb{R}^{2}$ given by

$$
\frac{\mathrm{d} x}{\mathrm{~d} t}=-b(t)+2 c(t) x+4 a(t) x^{2}+a(t) c_{0} y^{4}, \quad \frac{\mathrm{d} y}{\mathrm{~d} t}=(c(t)+4 a(t) x) y,
$$

where $a(t), b(t)$ and $c(t)$ are arbitrary $t$-dependent functions and $c_{0} \in\{0,1\}$. We call this system planar diffusion Riccati system, which is related to the $t$-dependent vector field

$$
X=a(t) X_{3}-b(t) X_{1}+c(t) X_{2},
$$

where

$$
X_{1}=\frac{\partial}{\partial x}, \quad X_{2}=2 x \frac{\partial}{\partial x}+y \frac{\partial}{\partial y}, \quad X_{3}=\left(4 x^{2}+c_{0} y^{4}\right) \frac{\partial}{\partial x}+4 x y \frac{\partial}{\partial y}
$$


satisfy the commutation relations

$$
\left[X_{1}, X_{2}\right]=2 X_{1}, \quad\left[X_{1}, X_{3}\right]=4 X_{2}, \quad\left[X_{2}, X_{3}\right]=2 X_{3} .
$$

Consequently, they span a Vessiot-Guldberg Lie algebra $V^{\mathrm{PDR}}$ isomorphic to $\mathfrak{s l}(2)$. Let us use again theorem 4.4 to determine to which one of the Lie algebras of table 1 is $V^{\mathrm{PDR}}$ diffeomorphic to. As the vector fields (6.8) satisfy (6.9), the Casimir tensor field $R$ (4.1) turns out to be

$$
R=c_{0} y^{4} \partial_{x} \otimes \partial_{x}-y^{2} \partial_{y} \otimes \partial_{y} \Rightarrow \mathcal{I}\left(V^{\mathrm{PDR}}\right)=-c_{0}
$$

Since the vector fields $X_{1}, X_{2}, X_{3}$ span a distribution of rank two, the theorem 4.4 applies. In view of the latter, the system (6.7) for $c_{0}=1$ is diffeomorphic to $\mathrm{I}_{4}$ and for $c_{0}=0$ to $\mathrm{I}_{5}$. Indeed, for $c_{0}=1$ the change of variables

$$
u:=2 x+y^{2}, \quad v:=2 x-y^{2}, \quad x=\frac{1}{2}(u+v), \quad y=\sqrt{u-v}
$$

maps (6.8) into a basis of $\mathrm{I}_{4}$ whose elements are proportional to those ones given table 1 after a relabeling of the variables. Writing the symplectic structure and the Hamiltonian functions given in table 1 in the initial coordinate system $\{x, y\}$, we obtain

$$
\omega=-\frac{\mathrm{d} x \wedge \mathrm{d} y}{y^{3}}, \quad h_{1}=\frac{1}{2 y^{2}}, \quad h_{2}=\frac{x}{y^{2}}, \quad h_{3}=2 \frac{x^{2}}{y^{2}}-\frac{1}{2} y^{2},
$$

which satisfy

$$
\left\{h_{1}, h_{2}\right\}_{\omega}=-2 h_{1}, \quad\left\{h_{1}, h_{3}\right\}_{\omega}=-4 h_{2}, \quad\left\{h_{2}, h_{3}\right\}_{\omega}=-2 h_{3} .
$$

For the case $c_{0}=0$, we have that the vector fields (6.8) form a basis of $\mathrm{I}_{5}$ (see table 1). Hence, their associated symplectic form and some corresponding Hamiltonian functions can easily be obtained from table 1. The main result of this section can be summarized as follows.

Proposition 6.3. The planar diffusion Riccati system (6.7) is a LH system of class $\mathrm{I}_{4}$ for $c_{0}=1$; and $\mathrm{I}_{5}$ for $c_{0}=0$.

\subsection{Equivalence among planar $\mathfrak{s l}(2)-\mathrm{LH}$ systems}

By taking into account the previous results, we are led to the following statement.

Theorem 6.4. The $\mathfrak{s l}(2)-L H$ systems (5.2), (6.1), (6.3), (6.5) and (6.7) are equivalent through local diffeomorphisms whenever they belong to the same class in table 1, that is,

- $\mathrm{P}_{2}$ : Milne-Pinney and Kummer-Schwarz equations for $c>0$ as well as complex Riccati equations with $t$-dependent real coefficients.

- $\mathrm{I}_{4}$ : Milne-Pinney and Kummer-Schwarz equations for $c<0$, coupled Riccati equations, split-complex Riccati equations and the planar diffusion Riccati system with $c_{0}=1$. All of them with t-dependent real coefficients.

- $\mathrm{I}_{5}$ : Milne-Pinney and Kummer-Schwarz equations for $c=0$ as well as dual-Study Riccati equations, planar diffusion Riccati systems with $c_{0}=0$ and the harmonic oscillator with t-dependent frequency. 
Only within each class, these systems are locally diffeomorphic and, therefore, there exists a local $t$-independent change of variables mapping one into another. For instance, there does not exist any diffeomorphism on $\mathbb{R}^{2}$ mapping the Milne-Pinney and Kummer-Schwarz equations with $c \neq 0$ to the harmonic oscillator with a $t$-dependent frequency as the latter is a LH system of class $\mathrm{I}_{5}$ and the previous ones do not. Our results also explain the existence of the known diffeomorphism mapping Kummer-Schwarz equations to Milne-Pinney equations provided their constant $c$ shares the same sign [22].

\section{Two-photon LH systems}

Let us study two different LH systems that belong to the same class $\mathrm{P}_{5}$ : systems related to $t$-dependent quadratic Hamiltonians and second-order Riccati equations in Hamiltonian form. As a consequence, we shall prove their equivalence through diffeomorphisms.

The elements of the basis $\left\{X_{1}, \ldots, X_{5}\right\}$ of $\mathrm{P}_{5}$ written in table 1 satisfy the Lie brackets

$$
\begin{aligned}
& {\left[X_{1}, X_{2}\right]=0, \quad\left[X_{1}, X_{3}\right]=X_{1}, \quad\left[X_{1}, X_{4}\right]=0, \quad\left[X_{1}, X_{5}\right]=X_{2},} \\
& {\left[X_{2}, X_{3}\right]=-X_{2}, \quad\left[X_{2}, X_{4}\right]=X_{1}, \quad\left[X_{2}, X_{5}\right]=0, \quad\left[X_{3}, X_{4}\right]=-2 X_{4},} \\
& {\left[X_{3}, X_{5}\right]=2 X_{5}, \quad\left[X_{4}, X_{5}\right]=-X_{3} \text {. }}
\end{aligned}
$$

Hence, they span a Lie algebra isomorphic to $\mathfrak{s l}(2) \ltimes \mathbb{R}^{2}$, where $\mathbb{R}^{2} \simeq\left\langle X_{1}, X_{2}\right\rangle$ and $\mathfrak{s l}(2) \simeq$ $\left\langle X_{3}, X_{4}, X_{5}\right\rangle$. Observe that this Lie algebra satisfies the conditions given by theorem 3.6, hence such vector fields are Hamiltonian relative to the Poisson bivector $\Lambda=X_{1} \wedge X_{2}=\partial_{x} \wedge \partial_{y}$ or, equivalently, the associated symplectic structure $\omega=\mathrm{d} x \wedge \mathrm{d} y$. This retrieves in an algebraic/geometric manner the result obtained in [13].

The corresponding Hamiltonian functions for $X_{1}, \ldots, X_{5}$ must be enlarged with a central generator $h_{0}=1$ giving rise to the centrally extended Lie algebra $\overline{\mathfrak{s l}(2) \ltimes \mathbb{R}^{2}}$ which is, in fact, isomorphic to the two-photon Lie algebra $\mathfrak{h}_{6}=\left\langle h_{1}, \ldots, h_{5}, h_{0}\right\rangle$ [30, 36]. That is why we shall call these systems two-photon LH systems. The commutation relations of this Lie algebra read

$$
\begin{array}{llll}
\left\{h_{1}, h_{2}\right\}_{\omega}=h_{0}, & \left\{h_{1}, h_{3}\right\}_{\omega}=-h_{1}, & \left\{h_{1}, h_{4}\right\}_{\omega}=0, & \left\{h_{1}, h_{5}\right\}_{\omega}=-h_{2}, \\
\left\{h_{2}, h_{3}\right\}_{\omega}=h_{2}, & \left\{h_{2}, h_{4}\right\}_{\omega}=-h_{1}, & \left\{h_{2}, h_{5}\right\}_{\omega}=0, & \left\{h_{3}, h_{4}\right\}_{\omega}=2 h_{4}, \\
\left\{h_{3}, h_{5}\right\}_{\omega}=-2 h_{5}, & \left\{h_{4}, h_{5}\right\}_{\omega}=h_{3}, & \left\{h_{0},\right\}_{\omega}=0 . &
\end{array}
$$

Notice that $\mathfrak{h}_{6} \simeq \mathfrak{s l}(2) \ltimes \mathfrak{h}_{3}$, where $\mathfrak{h}_{3} \simeq\left\langle h_{0}, h_{1}, h_{2}\right\rangle$ is the Heisenberg-Weyl Lie algebra and $\mathfrak{s l}(2) \simeq\left\langle h_{3}, h_{4}, h_{5}\right\rangle$. Since $\mathfrak{h}_{4} \simeq\left\langle h_{0}, h_{1}, h_{2}, h_{3}\right\rangle$ is the harmonic oscillator algebra (isomorphic to the LH algebra $\overline{\mathfrak{i s o}}(1,1)$ of the class $\left.I_{8}\right)$, we have the inclusions $\mathfrak{h}_{3} \subset \mathfrak{h}_{4} \subset \mathfrak{h}_{6}$.

\section{$7.1 \quad t$-dependent quadratic Hamiltonians}

We now study $t$-dependent quadratic Hamiltonians

$$
h(t, q, p)=\alpha(t) \frac{p^{2}}{2}+\beta(t) \frac{p q}{2}+\gamma(t) \frac{q^{2}}{2}+\delta(t) p+\epsilon(t) q+\phi(t),
$$

where $\alpha(t), \beta(t), \gamma(t), \delta(t), \epsilon(t), \phi(t)$ are arbitrary real $t$-dependent functions [26]. As particular cases, (7.2) describes certain damped and/or dissipative harmonic oscillators [27], electric charges 
in monochromatic electric fields [26], etc. The corresponding Hamilton equations read

$$
\begin{aligned}
& \frac{\mathrm{d} q}{\mathrm{~d} t}=\frac{\partial h}{\partial p}=\alpha(t) p+\beta(t) \frac{q}{2}+\delta(t), \\
& \frac{\mathrm{d} p}{\mathrm{~d} t}=-\frac{\partial h}{\partial q}=-\left(\beta(t) \frac{p}{2}+\gamma(t) q+\epsilon(t)\right) .
\end{aligned}
$$

This system has an associated $t$-dependent vector field

$$
X=\delta(t) X_{1}-\epsilon(t) X_{2}+\frac{\beta(t)}{2} X_{3}+\alpha(t) X_{4}-\gamma(t) X_{5},
$$

where $X_{1}, \ldots, X_{5}$ are, up to a trivial change of variables $x:=q$ and $y:=p$, the vector fields of the basis of $\mathrm{P}_{5}$ given in table 1. Hence, their Hamiltonian functions with respect to the symplectic structure $\omega=\mathrm{d} q \wedge \mathrm{d} p$ can easily be obtained from table 1 .

\subsection{Second-order Riccati equation}

Second-order Riccati equations, which were recently studied using the theory of Lie systems in [28], read

$$
\frac{\mathrm{d}^{2} x}{\mathrm{~d} t^{2}}+\left(f_{0}(t)+f_{1}(t) x\right) \frac{\mathrm{d} x}{\mathrm{~d} t}+c_{0}(t)+c_{1}(t) x+c_{2}(t) x^{2}+c_{3}(t) x^{3}=0
$$

with

$$
f_{1}(t)=3 \sqrt{c_{3}(t)}, \quad f_{0}(t)=\frac{c_{2}(t)}{\sqrt{c_{3}(t)}}-\frac{1}{2 c_{3}(t)} \frac{\mathrm{d} c_{3}(t)}{\mathrm{d} t}, \quad c_{3}(t)>0,
$$

where $c_{0}(t), c_{1}(t), c_{2}(t)$ are arbitrary $t$-dependent functions and $c_{3}(t)$ is a non-negative function. This differential equation arises by reducing third-order linear differential equations through a dilation symmetry and a $t$-reparametrization [29].

The key point is that a quite general family of second-order Riccati equations (7.4) admits a $t$-dependent Hamiltonian (see [28, 29]) given by

$$
h(t, x, p)=-2 \sqrt{-p}-p\left(a_{0}(t)+a_{1}(t) x+a_{2}(t) x^{2}\right), \quad p<0,
$$

where $a_{0}(t), a_{1}(t), a_{2}(t)$ are certain functions related to the $t$-dependent coefficients of (7.4). The corresponding Hamilton equations are

$$
\begin{aligned}
& \frac{\mathrm{d} x}{\mathrm{~d} t}=\frac{\partial h}{\partial p}=\frac{1}{\sqrt{-p}}-a_{0}(t)-a_{1}(t) x-a_{2}(t) x^{2}, \\
& \frac{\mathrm{d} p}{\mathrm{~d} t}=-\frac{\partial h}{\partial x}=p\left(a_{1}(t)+2 a_{2}(t) x\right),
\end{aligned}
$$

and the associated $t$-dependent vector field has the expression

$$
X=Y_{1}-a_{0}(t) Y_{2}-a_{1}(t) Y_{3}-a_{2}(t) Y_{4}
$$

where

$$
Y_{1}=\frac{1}{\sqrt{-p}} \frac{\partial}{\partial x}, \quad Y_{2}=\frac{\partial}{\partial x}, \quad Y_{3}=x \frac{\partial}{\partial x}-p \frac{\partial}{\partial p}, \quad Y_{4}=x^{2} \frac{\partial}{\partial x}-2 x p \frac{\partial}{\partial p} .
$$


Another vector field

$$
Y_{5}=\frac{x}{\sqrt{-p}} \frac{\partial}{\partial x}+2 \sqrt{-p} \frac{\partial}{\partial p}
$$

is required in order to close a Lie algebra $V^{\mathrm{SR}}$, whose non-vanishing commutation relations read

$$
\begin{array}{llll}
{\left[Y_{1}, Y_{3}\right]=\frac{1}{2} Y_{1},} & {\left[Y_{1}, Y_{4}\right]=Y_{5},} & {\left[Y_{2}, Y_{3}\right]=Y_{2},} & {\left[Y_{2}, Y_{4}\right]=2 Y_{3},} \\
{\left[Y_{2}, Y_{5}\right]=Y_{1},} & {\left[Y_{3}, Y_{4}\right]=Y_{4},} & {\left[Y_{3}, Y_{5}\right]=\frac{1}{2} Y_{5} .} &
\end{array}
$$

Hence, $V^{\mathrm{SR}} \simeq \mathfrak{s l}(2) \ltimes \mathbb{R}^{2}$ where $\mathbb{R}^{2} \simeq\left\langle Y_{1}, Y_{5}\right\rangle$ and $\mathfrak{s l}(2) \simeq\left\langle Y_{2}, Y_{3}, Y_{4}\right\rangle$.

Next, observe that $I=\left\langle Y_{1}, Y_{5}\right\rangle$ is an ideal of $V^{\mathrm{SR}}$ such that $I \wedge I \neq\{0\}$ and that it can be proven that all elements of $V^{\mathrm{SR}}$ act on $I$ as traceless operators. Therefore, in view of theorem [3.6. this Lie algebra consists of Hamiltonian vector fields with respect to the Poisson bivector $\Lambda:=\frac{1}{2} Y_{1} \wedge Y_{5}=\partial_{x} \wedge \partial_{p}$ or, equivalently, to the symplectic structure $\omega=\mathrm{d} x \wedge \mathrm{d} p$.

The Hamiltonian functions corresponding to the vector fields (7.6) and (7.7) turn out to be

$$
\tilde{h}_{1}=-2 \sqrt{-p}, \quad \tilde{h}_{2}=p, \quad \tilde{h}_{3}=x p, \quad \tilde{h}_{4}=x^{2} p, \quad \tilde{h}_{5}=-2 x \sqrt{-p},
$$

which span along with $\tilde{h}_{0}=1$ a Lie algebra of functions isomorphic to the two-photon Lie algebra $\mathfrak{h}_{6}$ with non-vanishing Lie brackets given by

$$
\begin{array}{llll}
\left\{\tilde{h}_{1}, \tilde{h}_{3}\right\}_{\omega}=-\frac{1}{2} \tilde{h}_{1}, & \left\{\tilde{h}_{1}, \tilde{h}_{4}\right\}_{\omega}=-\tilde{h}_{5}, \quad\left\{\tilde{h}_{1}, \tilde{h}_{5}\right\}_{\omega}=2 \tilde{h}_{0}, \quad\left\{\tilde{h}_{2}, \tilde{h}_{3}\right\}_{\omega}=-\tilde{h}_{2}, \\
\left\{\tilde{h}_{2}, \tilde{h}_{4}\right\}_{\omega}=-2 \tilde{h}_{3}, \quad\left\{\tilde{h}_{2}, \tilde{h}_{5}\right\}_{\omega}=-\tilde{h}_{1}, \quad\left\{\tilde{h}_{3}, \tilde{h}_{4}\right\}_{\omega}=-\tilde{h}_{4}, \quad\left\{\tilde{h}_{3}, \tilde{h}_{5}\right\}_{\omega}=-\frac{1}{2} \tilde{h}_{5} .
\end{array}
$$

Indeed, it can be seen that the functions

$$
\widehat{h}_{1}=-\frac{1}{\sqrt{2}} \tilde{h}_{5}, \quad \widehat{h}_{2}=\frac{1}{\sqrt{2}} \tilde{h}_{1}, \quad \widehat{h}_{3}=-2 \tilde{h}_{3}, \quad \widehat{h}_{4}=-\tilde{h}_{4}, \quad \widehat{h}_{5}=\tilde{h}_{2}, \quad \widehat{h}_{0}=\tilde{h}_{0}
$$

close the same commutation relations that the basis of $\mathfrak{h}_{6}$ given in (7.1). The main results of this section are then summarized as follows.

Proposition 7.1. The Hamilton equations (7.3) and (7.5) for t-dependent quadratic Hamiltonians and second-order Riccati equations (7.4), respectively, determine LH systems of class $\mathrm{P}_{5}$ with LH algebras isomorphic to the two-photon one $\mathfrak{h}_{6}$. Consequently all of these systems are locally diffeomorphic.

\section{Projective Schrödinger equations on $\mathbb{C P}^{1}$}

Let us describe a new application of LH systems on the plane. Consider the Schrödinger equations on $\mathbb{C}^{2}$ given by

$$
\mathrm{i} \frac{\mathrm{d}}{\mathrm{d} t}\left[\begin{array}{c}
\Psi_{1} \\
\Psi_{2}
\end{array}\right]=\left[\begin{array}{cc}
\lambda_{1}(t) & \beta(t) \\
\bar{\beta}(t) & \lambda_{2}(t)
\end{array}\right]\left[\begin{array}{c}
\Psi_{1} \\
\Psi_{2}
\end{array}\right]
$$

where $\lambda_{1}(t), \lambda_{2}(t)$ are arbitrary $t$-dependent real functions, $\beta(t)$ is an arbitrary $t$-dependent complex function and we assume $\hbar=1$ for simplicity. Let us construct the projection of this $t$ dependent Schrödinger equation onto the projective space $\mathbb{C}_{\times}^{2} \backslash \mathbb{C}^{\times} \simeq \mathbb{C P}^{1}$, where $\mathbb{C}^{\times}:=\mathbb{C}-\{0\}$ and $\mathbb{C}_{\times}^{2}=\mathbb{C}^{2} \backslash\{(0,0)\}$. Observe that $\left(\Psi_{1}, \Psi_{2}\right),\left(\Phi_{1}, \Phi_{2}\right) \in \mathbb{C}_{\times}^{2}$ belong to the same equivalence class of $\mathbb{C P}^{1}$, a so-called ray, if and only if $\Psi_{1} \Phi_{2}-\Phi_{1} \Psi_{2}=0$. Hence, elements $\left(\Psi_{1}, \Psi_{2}\right) \in U_{1}:=$ $\mathbb{C} \times \mathbb{C}^{\times}$belonging to the same ray give rise to the same complex number $\Psi_{1} \Psi_{2}^{-1} \in \mathbb{C}$. Using this, we can introduce a well-defined local coordinate system $\pi_{1}:\left[\left(\Psi_{1}, \Psi_{2}\right)\right] \in U_{1} \subset \mathbb{C P}^{1} \mapsto z:=$ 
$\Psi_{1} \Psi_{2}^{-1} \in \mathbb{C} \simeq \mathbb{R}^{2}$. Similarly, a second coordinate system can be defined on $U_{2}:=\mathbb{C}^{\times} \times \mathbb{C}$. A simple calculation shows that the projection of (8.1) under $\pi_{1}$ becomes

$$
\mathrm{i} \frac{\mathrm{d} z}{\mathrm{~d} t}=\beta(t)-\bar{\beta}(t) z^{2}+\left(\lambda_{1}(t)-\lambda_{2}(t)\right) z .
$$

Writing $z=x+\mathrm{i} y$ and $\beta(t)=\beta_{x}(t)+\mathrm{i} \beta_{y}(t)$, we obtain

$$
\begin{aligned}
& \frac{\mathrm{d} x}{\mathrm{~d} t}=-\beta_{x}(t) 2 x y+\beta_{y}(t)\left(x^{2}-y^{2}+1\right)+\left(\lambda_{1}(t)-\lambda_{2}(t)\right) y, \\
& \frac{\mathrm{d} y}{\mathrm{~d} t}=\beta_{x}(t)\left(x^{2}-y^{2}-1\right)+\beta_{y}(t) 2 x y-\left(\lambda_{1}(t)-\lambda_{2}(t)\right) x .
\end{aligned}
$$

This system of differential equations describes the integral curves of the $t$-dependent vector field $X=-\beta_{x}(t) X_{3}+\beta_{y}(t) X_{2}+\left(\lambda_{1}(t)-\lambda_{2}(t)\right) X_{1}$, where

$$
X_{1}=y \frac{\partial}{\partial x}-x \frac{\partial}{\partial y}, \quad X_{2}=\left(1+x^{2}-y^{2}\right) \frac{\partial}{\partial x}+2 x y \frac{\partial}{\partial y}, \quad X_{3}=2 x y \frac{\partial}{\partial x}+\left(1+y^{2}-x^{2}\right) \frac{\partial}{\partial y} .
$$

These are exactly the vector fields appearing in the Lie algebra $\mathrm{P}_{3}$ of table 1. So, they span a Lie algebra of vector fields isomorphic to $\mathfrak{s o}(3) \simeq \mathfrak{s u}(2)$ and $X_{1}, X_{2}, X_{3}$ are Hamiltonian with respect to the symplectic form given in table 1, namely

$$
\omega=\frac{\mathrm{d} x \wedge \mathrm{d} y}{\left(1+x^{2}+y^{2}\right)^{2}} .
$$

As a consequence of our results and [13, Theorem 4.4], this is the only symplectic structure on the projective space, up to a constant multiplicative factor, turning (8.2) into a LH system for arbitrary complex function $\beta(t)$, and real functions $\lambda_{1}(t)$ and $\lambda_{2}(t)$.

\section{$9 \quad$ Planar $\mathfrak{h}_{2}-\mathrm{LH}$ systems}

We now focus on the Lie algebra $\mathrm{I}_{14 A} \simeq \mathbb{R} \ltimes \mathbb{R}^{r}$ with $r=1$ of table 1, so with a basis of vector fields $X_{1}=\partial_{x}, X_{2}=\eta_{1}(x) \partial_{y}$ with $\eta_{1}(x) \notin\langle 1\rangle$. If we require that these close a non-Abelian Lie algebra and we choose, with no loss of generality, that $\left[X_{1}, X_{2}\right]=X_{2}$, then $\eta_{1}(x)=\mathrm{e}^{x}$ up to an irrelevant proportional non-zero constant, that is

$$
X_{1}=\frac{\partial}{\partial x}, \quad X_{2}=\mathrm{e}^{x} \frac{\partial}{\partial y}, \quad\left[X_{1}, X_{2}\right]=X_{2},
$$

and we denote $\left\langle X_{1}, X_{2}\right\rangle:=\mathfrak{h}_{2} \simeq \mathbb{R} \ltimes \mathbb{R} \simeq \mathrm{I}_{14 A}^{r=1}$. This is a Vessiot-Guldberg Lie algebra of Hamiltonian vector fields relative to the symplectic form $\omega=\mathrm{d} x \wedge \mathrm{d} y$. Hence, we can choose

$$
h_{1}=y, \quad h_{2}=-\mathrm{e}^{x}, \quad\left\{h_{1}, h_{2}\right\}_{\omega}=-h_{2} .
$$

In the following, we show, as a new result, that $\mathfrak{h}_{2}$ underlies the complex Bernoulli differential equations with $t$-dependent real coefficients and we relate this result with other known ones concerning generalised Buchdahl equations and $t$-dependent Lotka-Volterra systems. It is remarkable that Cayley-Klein Riccati equations (5.2) with $a_{2}(t)=0$ are $\mathfrak{h}_{2}$-LH systems as well. Finally, we prove that all of these systems belong to the same class $I_{14 A}^{r=1} \simeq \mathfrak{h}_{2}$. 


\subsection{Complex Bernoulli differential equation with $t$-dependent real coefficients}

Let us restrict ourselves to studying the complex differential Bernoulli equation (1.3) of the form

$$
\frac{\mathrm{d} z}{\mathrm{~d} t}=a_{1}(t) z+a_{2}(t) z^{n}, \quad n \notin\{0,1\},
$$

with $a_{1}(t), a_{2}(t)$ being arbitrary real functions. It can proved that the planar nonautonomous Bernoulli-like equations appearing in [52, p. 197] when solving equations in the Abel chains can be mapped into a particular case of the above system by means of an appropriate diffeomorphism.

The equation (9.3) can be studied in terms of the $t$-dependent vector field $X=a_{1}(t) X_{0}+$ $a_{2}(t) X_{2}$, where we recall that the vector fields $X_{0}$ and $X_{2}$ are given in (1.5) and satisfy

$$
\left[X_{0}, X_{2}\right]=(n-1) X_{2},
$$

which is isomorphic to $\mathfrak{h}_{2}$. In the GKO classification [12, 13] there is just one Lie algebra isomorphic to $\mathfrak{h}_{2}$ whose vector fields are not proportional at each point: $\mathrm{I}_{14 A}$ with $r=1$. So, $\left\langle X_{0}, X_{2}\right\rangle$ is a Lie algebra of Hamiltonian vector fields in view of the results of table 1 ,

In order to study this system, we provide the change of variables

$$
x:=\ln \left|\frac{r^{n-1}}{\sin [\theta(n-1)]}\right|, \quad y:=-\frac{\operatorname{cotg}[\theta(n-1)]}{n-1}
$$

mapping the vector fields $\bar{X}_{1}=X_{0} /(n-1)$ and $X_{2}$ into (9.1). The symplectic form and Hamiltonian functions for $X_{0}$ and $X_{2}$ in the initial variables $r, \theta$ can be obtained from $\omega=\mathrm{d} x \wedge \mathrm{d} y$ and (9.2) by inverting the above change of variables.

\subsection{Generalized Buchdahl equations}

The generalized Buchdahl equations, appearing in the study of relativistic fluids [31, 32] and whose properties have been studied through a Lagrangian approach in [33], are the second-order differential equations given by

$$
\frac{\mathrm{d}^{2} x}{\mathrm{~d} t^{2}}=a(x)\left(\frac{\mathrm{d} x}{\mathrm{~d} t}\right)^{2}+b(t) \frac{\mathrm{d} x}{\mathrm{~d} t}
$$

for arbitrary functions $a(x)$ and $b(t)$. If we set $y:=\mathrm{d} x / \mathrm{d} t$, we find the first-order system of differential equations

$$
\frac{\mathrm{d} x}{\mathrm{~d} t}=y, \quad \frac{\mathrm{d} y}{\mathrm{~d} t}=a(x) y^{2}+b(t) y,
$$

which is associated with the $t$-dependent vector field $X=X_{2}+b(t) X_{1}$, where

$$
X_{1}=y \frac{\partial}{\partial y}, \quad X_{2}=y \frac{\partial}{\partial x}+a(x) y^{2} \frac{\partial}{\partial y},
$$

satisfy $\left[X_{1}, X_{2}\right]=X_{2}$. These vector fields span a Lie algebra diffeomorphic to $\mathrm{I}_{14 A}^{r=1} \simeq \mathfrak{h}_{2}$ and (9.4) becomes a LH system. The corresponding symplectic form and Hamiltonian functions can be found in [13]. 


\section{$9.3 \quad t$-dependent Lotka-Volterra systems}

Finally, consider the particular Lotka-Volterra systems [34, 35] given by

$$
\frac{\mathrm{d} x}{\mathrm{~d} t}=a x-g(t)(x-a y) x, \quad \frac{\mathrm{d} y}{\mathrm{~d} t}=a y-g(t)(b x-y) y, \quad a \neq 0,
$$

where $g(t)$ determines the variation of the seasons, while $a$ and $b$ are constants describing the interactions among the species. System (9.5) is associated with the $t$-dependent vector field $X=X_{1}+g(t) X_{2}$ where

$$
X_{1}=a x \frac{\partial}{\partial x}+a y \frac{\partial}{\partial y}, \quad X_{2}=-(x-a y) x \frac{\partial}{\partial x}-(b x-y) y \frac{\partial}{\partial y},
$$

satisfy

$$
\left[X_{1}, X_{2}\right]=a X_{2} .
$$

Hence, (9.5) is a Lie system. Moreover, it has been proven in [13] that, except for the case with $a=b=1$, this is also a LH system belonging to the family $\mathrm{I}_{14 A}^{r=1} \simeq \mathfrak{h}_{2}$. The symplectic form and the Hamiltonian functions for $X_{1}$ and $X_{2}$ can be found in [13].

Hence, we conclude this section with the following statement.

Proposition 9.1. The complex Bernoulli differential equation (9.3), the generalized Buchdahl equations (9.4) and the $t$-dependent Lotka-Volterra systems (9.5) (with the exception of $a=b=$ 1) are LH systems with a Vessiot-Guldberg Lie algebra diffeomorphic to $I_{14 A}^{r=1} \simeq \mathfrak{h}_{2}$ in table 1 . Thus all of these systems are locally diffeomorphic.

\section{Constants of motion and superposition rules}

As commented in the introduction, one of the most outstanding properties of LH systems is that their superposition rules (which exist for any Lie system) can be obtained by applying the coalgebra approach recently introduced in [19] in an easier way than by applying traditional methods [2, 9]. Essentially, for any LH system, this procedure requires to endow the LH algebra with a coalgebra structure which is provided by a trivial (non-deformed) coproduct map. Next, $t$-independent constants of motion can be obtained from the $m$ th-order coproduct of a non-trivial Casimir invariant and the corresponding superposition rule can then be worked out by starting from such constants of motion.

In this section we firstly provide the constants of motion for the LH algebras displayed in table 1 and, secondly, we use them in the construction of superposition rules for planar LH systems. In this respect, we recall that, to the best of our knowledge, this coalgebra approach has only been applied in [19] to planar $\mathfrak{s l}(2)$ and $\mathfrak{s o}(3)$-LH systems obtaining their superposition rules. Therefore, we shall restrict ourselves to studying the remaining LH systems here considered, that is, LH systems of classes $\mathrm{P}_{1}, \mathrm{P}_{5}$ and $\mathrm{I}_{14 A}^{r=1}$ (via the class $\mathrm{I}_{8}$ ). Hereafter, we shall omit most technical details, which can be found in [19], and so we shall briefly summarize the necessary essential tools so as to have a self-contained paper.

\subsection{Constants of motion}

Assuming the notation introduced in section 3, we consider a Lie algebra $\mathfrak{g}$ spanned by the generators $\left\{v_{1}, \ldots, v_{l}\right\}$ and its corresponding symmetric algebra $S(\mathfrak{g})$ understood as a Poisson 
algebra. Then $S(\mathfrak{g})$ can always be endowed with a Poisson coalgebra structure by introducing the (non-deformed) coproduct map $\Delta: S(\mathfrak{g}) \rightarrow S(\mathfrak{g}) \otimes S(\mathfrak{g})$ defined by

$$
\Delta(v)=v \otimes 1+1 \otimes v, \quad \forall v \in \mathfrak{g} \subset S(\mathfrak{g}),
$$

which is a Poisson algebra homomorphism. The coproduct $\Delta \equiv \Delta^{(2)}$ can be extended to a third-order coproduct $\Delta^{(3)}: S(\mathfrak{g}) \rightarrow S(\mathfrak{g}) \otimes S(\mathfrak{g}) \otimes S(\mathfrak{g}) \equiv S^{(3)}(\mathfrak{g})$ by means of the coassociatity condition [53], $\Delta^{(3)}:=(\Delta \otimes \mathrm{Id}) \circ \Delta=(\operatorname{Id} \otimes \Delta) \circ \Delta$, namely

$$
\Delta^{(3)}(v)=v \otimes 1 \otimes 1+1 \otimes v \otimes 1+1 \otimes 1 \otimes v, \quad \forall v \in \mathfrak{g} \subset S(\mathfrak{g}) .
$$

And the $m$ th-order coproduct map $\Delta^{(m)}: S(\mathfrak{g}) \rightarrow S^{(m)}(\mathfrak{g})$ can be defined, recursively, as

$$
\Delta^{(m)}:=(\overbrace{\operatorname{Id} \otimes \ldots \otimes \operatorname{Id}}^{(m-2)-\text {-times }} \otimes \Delta^{(2)}) \circ \Delta^{(m-1)}, \quad m \geq 3,
$$

which, clearly, is also a Poisson algebra homomorphism.

Let $X$ be a LH system related to a LH algebra $\mathcal{H}_{\Lambda}$ spanned by the linearly independent Hamiltonian functions $\left\{h_{1}, \ldots, h_{l}\right\}$ and let $D$ be the Poisson algebra morphism $D: S(\mathfrak{g}) \rightarrow$ $C^{\infty}(M)$ induced by extending to $S(\mathfrak{g})$ the injection $\iota: \mathfrak{g} \hookrightarrow \mathcal{H}_{\Lambda} \subset C^{\infty}(M)$, with $\phi\left(v_{i}\right)=h_{i}$ for $i=1, \ldots, l$. By extension, we can construct a family of Poisson algebra morphisms $D^{(m)}$ : $S^{(m)}(\mathfrak{g}) \rightarrow C^{\infty}(M)^{(m)} \subset C^{\infty}\left(M^{m}\right)$. If $C$ is a polynomial Casimir of the Poisson algebra $S(\mathfrak{g})$, say $C=C\left(v_{1}, \ldots, v_{l}\right)$, then $D(C)$ is a constant of motion for $X$ and the functions

$$
F^{(k)}\left(h_{1}, \ldots, h_{l}\right)=D^{(k)}\left[\Delta^{(k)}\left(C\left(v_{1}, \ldots, v_{l}\right)\right)\right], \quad k=2, \ldots, m,
$$

are $t$-independent constants of motion for the diagonal prolongation $\widetilde{X}$ to the $m$ th manifold $M^{m}$, namely if $X=\sum_{i=1}^{n} X^{i}(x) \partial / \partial x^{i}$, then

$$
\widetilde{X}=\sum_{a=1}^{m} \sum_{i=1}^{n} X^{i}\left(x_{(a)}\right) \frac{\partial}{\partial x_{(a)}^{i}},
$$

where $\left(x_{(1)}, \ldots, x_{(m)}\right) \in M^{m}$ (see [9] for details on diagonal prolongations). Observe also that each $F^{(k)}$ can naturally be considered as a function of $C^{\infty}\left(M^{m}\right)$ for every $m \geq k$.

If all the $F^{(k)}$ are non-constant functions, then they form a set of $(m-1)$ functionally independent functions in involution in $C^{\infty}\left(M^{m}\right)$ (cf. theorem 26 in [19]). Furthermore, from the functions $F^{(k)}$ other constants of motion can be obtained in the form

$$
F_{i j}^{(k)}=S_{i j}\left(F^{(k)}\right), \quad 1 \leq i<j \leq k, \quad k=2, \ldots, m,
$$

where $S_{i j}$ is the permutation of variables $x_{(i)} \leftrightarrow x_{(j)}$ on $M^{m}$. Indeed, since $\tilde{X}$ is invariant under the permutations $x_{(i)} \leftrightarrow x_{(j)}$, then the $F_{i j}^{(k)}$ are also $t$-independent constants of motion for the diagonal prolongations $\widetilde{X}$ to $M^{m}$.

Let us now illustrate the previous procedure by considering a LH system $X$ taking values in $\mathrm{P}_{1} \simeq \mathfrak{i s o}(2)$. Using the basis $\left\{X_{1}, X_{2}, X_{3}\right\}$ of $\mathrm{P}_{1}$ given in table 1, we have

$$
\left[X_{1}, X_{2}\right]=0, \quad\left[X_{1}, X_{3}\right]=-X_{2}, \quad\left[X_{2}, X_{3}\right]=X_{1} .
$$

The corresponding LH algebra is isomorphic to $\overline{\mathfrak{i s o}}(2)$ and it admits a basis $\left\{h_{1}, h_{2}, h_{3}, h_{0}\right\}$ (see table 1) satisfying commutation relations

$$
\left\{h_{1}, h_{2}\right\}_{\omega}=h_{0}, \quad\left\{h_{1}, h_{3}\right\}_{\omega}=h_{2}, \quad\left\{h_{2}, h_{3}\right\}_{\omega}=-h_{1}, \quad\left\{h_{0}, \cdot\right\}_{\omega}=0,
$$


with respect to the canonical symplectic structure $\omega=\mathrm{d} x \wedge \mathrm{d} y$. The symmetric Poisson algebra $S(\overline{\mathfrak{i s o}}(2))$ of $\overline{\mathfrak{i s o}}(2)$, where $\left\{v_{1}, v_{2}, v_{3}, v_{0}\right\}$ is a basis of $\overline{\mathfrak{i s o}}(2)$ fulfilling the commutation rules (10.4), has a non-trivial Casimir invariant given by

$$
C=v_{3} v_{0}-\frac{1}{2}\left(v_{1}^{2}+v_{2}^{2}\right)
$$

From it, we obtain a trivial constant of motion on the variables $(x, y) \equiv\left(x_{1}, y_{1}\right)$ :

$$
\begin{aligned}
F & =D(C)=\phi\left(v_{3}\right) \phi\left(v_{0}\right)-\frac{1}{2}\left(\phi^{2}\left(v_{1}\right)+\phi^{2}\left(v_{2}\right)\right) \\
& =h_{3}\left(x_{1}, y_{1}\right) h_{0}\left(x_{1}, y_{1}\right)-\frac{1}{2}\left[h_{1}^{2}\left(x_{1}, y_{1}\right)+h_{2}^{2}\left(x_{1}, y_{1}\right)\right]=\frac{1}{2}\left(x_{1}^{2}+y_{1}^{2}\right) \times 1-\frac{1}{2}\left(y_{1}^{2}+x_{1}^{2}\right)=0 .
\end{aligned}
$$

Nevertheless, once the coalgebra structure is introduced in $S(\overline{\mathfrak{i s o}}(2))$ through the coproduct (10.1), the functions (10.2) are no longer trivial ones and we find, for $m=3$ (so $k=2,3$ ), that

$$
\begin{aligned}
F^{(2)}= & D^{(2)}(\Delta(C))=\left(h_{3}\left(x_{1}, y_{1}\right)+h_{3}\left(x_{2}, y_{2}\right)\right)\left(h_{0}\left(x_{1}, y_{1}\right)+h_{0}\left(x_{2}, y_{2}\right)\right) \\
& \quad-\frac{1}{2}\left[\left(\left(h_{1}\left(x_{1}, y_{1}\right)+h_{1}\left(x_{2}, y_{2}\right)\right)^{2}+\left(h_{2}\left(x_{1}, y_{1}\right)+h_{2}\left(x_{2}, y_{2}\right)\right)^{2}\right]\right. \\
= & \frac{1}{2}\left[\left(x_{1}-x_{2}\right)^{2}+\left(y_{1}-y_{2}\right)^{2}\right] \\
F^{(3)}= & D^{(3)}(\Delta(C))=\sum_{i=1}^{3} h_{3}\left(x_{i}, y_{i}\right) \sum_{j=1}^{3} h_{0}\left(x_{j}, y_{j}\right) \\
& \quad-\frac{1}{2}\left[\left(\sum_{i=1}^{3} h_{1}\left(x_{i}, y_{i}\right)\right)^{2}+\left(\sum_{i=1}^{3} h_{2}\left(x_{i}, y_{i}\right)\right)^{2}\right] \\
= & \frac{1}{2} \sum_{1 \leq i<j}^{3}\left[\left(x_{i}-x_{j}\right)^{2}+\left(y_{i}-y_{j}\right)^{2}\right] .
\end{aligned}
$$

For $k=2$, the constants (10.3) read

$$
\begin{aligned}
& F_{12}^{(2)}=S_{12}\left(F^{(2)}\right) \equiv F^{(2)}, \quad F_{13}^{(2)}=S_{13}\left(F^{(2)}\right)=\frac{1}{2}\left[\left(x_{3}-x_{2}\right)^{2}+\left(y_{3}-y_{2}\right)^{2}\right], \\
& F_{23}^{(2)}=S_{23}\left(F^{(2)}\right)=\frac{1}{2}\left[\left(x_{1}-x_{3}\right)^{2}+\left(y_{1}-y_{3}\right)^{2}\right]
\end{aligned}
$$

so that $F^{(3)}=F_{12}^{(2)}+F_{13}^{(2)}+F_{23}^{(2)}$. Observe that $F^{(2)}$ and $F^{(3)}$ satisfy that $\left.\partial\left(F^{(2)}, F^{(3)}\right) / \partial_{\left(x_{1}, y_{1}\right)}\right) \neq$ 0 . We can also choose two other functions among the set $\left\{F^{(2)}, F_{13}^{(2)}, F_{23}^{(2)}\right\}$ satisfying this condition. This will be important for deriving superposition rules for LH systems of class $\mathrm{P}_{1}$.

We display in table2 the first non-trivial invariants $F^{(k)}$ for each of the LH algebras of table1, except for the trivial Abelian cases $\mathrm{I}_{1} \simeq \mathbb{R}$ and $\mathrm{I}_{12} \simeq \mathbb{R}^{r+1}$. This is $F^{(3)}$ for the classes $\mathrm{P}_{5}$ and $\mathrm{I}_{16}$, and $F^{(2)}$ for the remaining ones. Notice that we have expressed the corresponding Casimir $C$ in terms of $h_{i}$, instead of $v_{i}$, to facilitate the reading with respect to table 1 . We remark that the LH algebras of the classes $\mathrm{I}_{14 A}, \mathrm{I}_{14 B}$ and $\mathrm{I}_{16}$ have no non-trivial invariant for $r=1$ and that for $\mathrm{I}_{14 A}$ and $\mathrm{I}_{14 B}$ a choice of the functions $\eta_{j}(x)$ must be performed. We have worked out this latter case with $r=2$ and the specific functions $\eta_{j}(x)$ indicated in table 2. In all the cases for which $F^{(2)}$ is not a trivial constant, it is found that $F^{(3)}=F_{12}^{(2)}+F_{13}^{(2)}+F_{23}^{(2)}+$ constant.

The results of table 2 are important due to the fact that allow us to construct a superposition rule for $X$. More precisely, let $X$ be a LH system on $\mathbb{R}^{2}$ admitting a Vessiot-Guldberg Lie algebra of Hamiltonian vector fields $V$ with basis $\left\{X_{1}, \ldots, X_{l}\right\}$. Then, a set $I_{1}, \ldots, I_{n} \in C^{\infty}\left(M^{m}\right)$ of functionally independent constants of motion for the diagonal prolongation $\widetilde{X}$ on $M^{m}$, with $m_{0}:=m-1$ being such that $\widetilde{X}_{1} \wedge \ldots \wedge \widetilde{X}_{l} \neq 0$ for the prolongations to $M^{m_{0}}$, enables us to determine a superposition rule for $X$ provided $\partial\left(I_{1}, \ldots, I_{n}\right) / \partial\left(x_{(0)}^{1}, \ldots, x_{(0)}^{n}\right) \neq 0$ (see [9] for details). Indeed, the latter condition ensures that $x_{(0)}^{1}, \ldots, x_{(0)}^{n}$ can be expressed in terms of $n$-constants $k_{1}, \ldots, k_{n}$ and the remaining variables in $M^{m}$ by solving the equations $I_{1}=$ $k_{1}, \ldots, I_{n}=k_{n}$. This leads to a superposition rule for $X$. For Lie systems, $I_{1}, \ldots, I_{n}$ are usually obtained by solving a system of PDEs, whereas they can be derived algebraically for planar LH systems following table 2, Exemplifying this for several new superposition rules for LH systems on the plane will be the aim of following subsections. 
Table 2: Constants of motion for LH algebras of table 1,

\begin{tabular}{|c|c|c|}
\hline$\#$ & LH algebra & Casimir $C$ and invariants $F^{(k)}$ \\
\hline $\mathrm{P}_{1}$ & $\overline{\mathfrak{i s o}}(2)$ & $\begin{array}{l}C=h_{3} h_{0}-\frac{1}{2}\left(h_{1}^{2}+h_{2}^{2}\right) \\
F=0 \quad F^{(2)}=\frac{1}{2}\left[\left(x_{1}-x_{2}\right)^{2}+\left(y_{1}-y_{2}\right)^{2}\right]\end{array}$ \\
\hline $\mathrm{P}_{2}$ & $\mathfrak{s l}(2)$ & $\begin{array}{l}C=h_{1} h_{3}-h_{2}^{2} \\
F=1 \quad F^{(2)}=\frac{\left(x_{1}-x_{2}\right)^{2}+\left(y_{1}+y_{2}\right)^{2}}{y_{1} y_{2}}\end{array}$ \\
\hline $\mathrm{P}_{3}$ & $\mathfrak{s o}(3) \oplus \mathbb{R}$ & $\begin{array}{l}C=4 h_{1}^{2}+h_{2}^{2}+h_{3}^{2}+2 h_{1} h_{0} \\
F=0 \quad F^{(2)}=-\frac{\left(x_{1}-x_{2}\right)^{2}+\left(y_{1}-y_{2}\right)^{2}}{\left(1+x_{1}^{2}+y_{1}^{2}\right)\left(1+x_{2}^{2}+y_{2}^{2}\right)}\end{array}$ \\
\hline $\mathrm{P}_{5}$ & $\mathfrak{h}_{6} \simeq \overline{\mathfrak{s l}(2) \ltimes \mathbb{R}^{2}}$ & $\begin{array}{l}C=2\left(h_{1}^{2} h_{5}-h_{2}^{2} h_{4}-h_{1} h_{2} h_{3}\right)-h_{0}\left(h_{3}^{2}+4 h_{4} h_{5}\right) \\
F=0 \quad F^{(2)}=0 \quad F^{(3)}=\left[x_{1}\left(y_{2}-y_{3}\right)+x_{2}\left(y_{3}-y_{1}\right)+x_{3}\left(y_{1}-y_{2}\right)\right]^{2}\end{array}$ \\
\hline $\mathrm{I}_{4}$ & $\mathfrak{s l}(2)$ & $\begin{array}{l}C=h_{1} h_{3}-h_{2}^{2} \\
F=-\frac{1}{4} \quad F^{(2)}=-\frac{\left(x_{2}-y_{1}\right)\left(x_{1}-y_{2}\right)}{\left(x_{1}-y_{1}\right)\left(x_{2}-y_{2}\right)}\end{array}$ \\
\hline $\mathrm{I}_{5}$ & $\mathfrak{s l}(2)$ & $\begin{array}{l}C=h_{1} h_{3}-h_{2}^{2} \\
F=0 \quad F^{(2)}=\frac{\left(x_{1}-x_{2}\right)^{2}}{\left(2 y_{1} y_{2}\right)^{2}}\end{array}$ \\
\hline $\mathrm{I}_{8}$ & $\overline{\mathfrak{i s o}}(1,1)$ & $\begin{array}{l}C=h_{1} h_{2}+h_{3} h_{0} \\
F=0 \quad F^{(2)}=\left(x_{1}-x_{2}\right)\left(y_{1}-y_{2}\right)\end{array}$ \\
\hline $\mathrm{I}_{14 A}^{r=2}$ & $\mathbb{R} \ltimes \mathbb{R}^{2}$ & $\begin{array}{lc}C=h_{2} h_{3} & \eta_{1}(x)=\mathrm{e}^{x} \quad \eta_{2}(x)=\mathrm{e}^{-x} \\
F=-1 & F^{(2)}=-2\left[1+\cosh \left(x_{1}-x_{2}\right)\right]\end{array}$ \\
\hline $\mathrm{I}_{14 B}^{r=2}$ & $\overline{\mathbb{R} \ltimes \mathbb{R}^{2}}$ & $\begin{array}{l}C=h_{2}^{2}+2 h_{3} h_{0} \quad \eta_{2}(x)=x \\
F=0 \quad F^{(2)}=-\left(x_{1}-x_{2}\right)^{2}\end{array}$ \\
\hline $\mathrm{I}_{16}$ & $\overline{\mathfrak{h}_{2} \ltimes \mathbb{R}^{r+1}}$ & $\begin{array}{l}C=\frac{2 h_{2}^{3}+6 h_{2} h_{4} h_{0}+3 h_{5} h_{0}^{2}}{3 h_{0}^{2}\left(h_{2}^{2}+2 h_{4} h_{0}\right)^{3 / 2}} \quad r \geq 2 \\
F=\operatorname{indet} \quad F^{(2)}=0 \quad F^{(3)}=\frac{\left(x_{1}+x_{2}-2 x_{3}\right)\left(x_{1}+x_{3}-2 x_{2}\right)\left(x_{2}+x_{3}-2 x_{1}\right)}{54 \sqrt{2}\left(x_{1} x_{2}+x_{1} x_{3}+x_{2} x_{3}-x_{1}^{2}-x_{2}^{2}-x_{3}^{2}\right)^{3 / 2}}\end{array}$ \\
\hline
\end{tabular}

\subsection{Superposition rules for $\mathrm{LH}$ systems of class $\mathbf{P}_{1} \simeq \mathfrak{i s o}(2)$}

Consider a LH system $X$ with a Vessiot-Guldberg Lie algebra $\mathrm{P}_{1}$. It can be proved that $m_{0}=2$ in this case. Let us consider the three constants of motion (10.5) written as

$$
\begin{aligned}
& F^{(2)}=\frac{1}{2}\left[\left(x_{1}-x_{2}\right)^{2}+\left(y_{1}-y_{2}\right)^{2}\right]=\frac{1}{2} k_{1}^{2} \geq 0, \\
& F_{23}^{(2)}=\frac{1}{2}\left[\left(x_{1}-x_{3}\right)^{2}+\left(y_{1}-y_{3}\right)^{2}\right]=\frac{1}{2} k_{2}^{2} \geq 0, \\
& F_{13}^{(2)}=\frac{1}{2}\left[\left(x_{3}-x_{2}\right)^{2}+\left(y_{3}-y_{2}\right)^{2}\right]=\frac{1}{2} k_{3}^{2}>0 .
\end{aligned}
$$

We aim to express the general solution $\left(x_{1}(t), y_{1}(t)\right)$ of our LH system in terms of two different particular solutions $\left(x_{i}(t), y_{i}(t)\right)$, with $i=2,3$. This is obtained by starting from the two first equations (10.6). The resulting expressions can next be simplified by introducing the third 


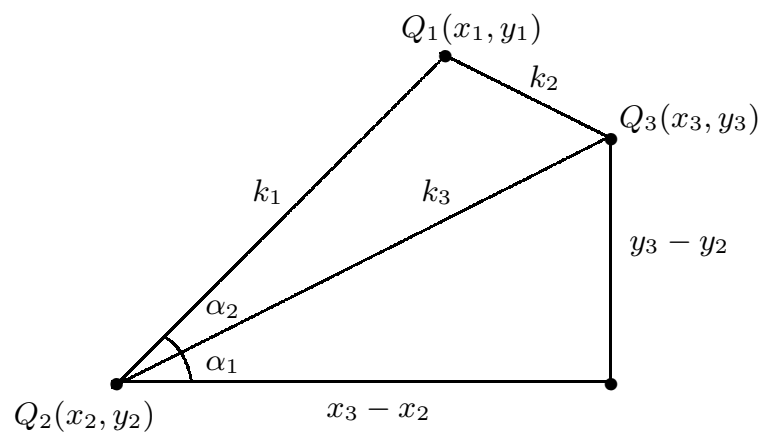

Figure 1: Geometrical description of the three constants of motion (10.6) as the sides of a triangle.

constant $k_{3}$, giving rise to

$$
\begin{aligned}
& x_{1}^{ \pm}\left(x_{2}, y_{2}, x_{3}, y_{3}, k_{1}, k_{2}, k_{3}\right)=x_{2}+\frac{k_{1}^{2}+k_{3}^{2}-k_{2}^{2}}{2 k_{3}^{2}}\left(x_{3}-x_{2}\right) \mp 2 A \frac{\left(y_{3}-y_{2}\right)}{k_{3}^{2}}, \\
& y_{1}^{ \pm}\left(x_{2}, y_{2}, x_{3}, y_{3}, k_{1}, k_{2}, k_{3}\right)=y_{2}+\frac{k_{1}^{2}+k_{3}^{2}-k_{2}^{2}}{2 k_{3}^{2}}\left(y_{3}-y_{2}\right) \pm 2 A \frac{\left(x_{3}-x_{2}\right)}{k_{3}^{2}},
\end{aligned}
$$

where $A$ is a constant given in terms of $k_{1}, k_{2}, k_{3}$ by

$$
A=\frac{1}{4} \sqrt{2\left(k_{1}^{2} k_{2}^{2}+k_{1}^{2} k_{3}^{2}+k_{2}^{2} k_{3}^{2}\right)-\left(k_{1}^{4}+k_{2}^{4}+k_{3}^{4}\right)} .
$$

We can assume with no loss of generality that $k_{1}, k_{2}, k_{3} \geq 0$. Since we assume $\left(x_{2}, y_{2}\right) \neq\left(x_{3}, y_{3}\right)$, we can set $k_{3}>0$. Therefore, the equations (10.7) comprise two cases (according to the signs \pm and $\mp$ ), which are well defined when the radicand of $A$ is non-negative, namely when $k_{i} \leq k_{j}+k_{l}$ for different $i, j, l$. Since $k_{3}=k_{3}\left(x_{2}, y_{2}, x_{3}, y_{3}\right)$, we can understand expressions (10.7) as a superposition rule $\Psi:\left(x_{2}, y_{2}, x_{3}, y_{3}, \pm k_{1}, k_{2}\right) \in U \subset \mathbb{R}^{4} \times\left(\mathbb{R} \times \mathbb{R}_{k_{2} \geq 0}\right) \mapsto\left(x_{1}^{ \pm}, y_{1}^{ \pm}\right) \in \mathbb{R}^{2}$. This enables us to express the general solution $\left(x_{1}(t), y_{1}(t)\right) \equiv(x(t), y(t))$ of $X$ in terms of two different particular solutions $\left(x_{2}(t), y_{2}(t)\right)$ and $\left(x_{3}(t), y_{3}(t)\right)$.

Alternatively, the constants of motion (10.6) and the derivation of the superposition rule (10.7) admit a geometrical approach. If $k_{1}, k_{2}, k_{3}$ are non-negative constants, these can be understood as the lengths of the segments $\overline{Q_{1} Q_{2}}, \overline{Q_{1} Q_{3}}$ and $\overline{Q_{2} Q_{3}}$ between the points $Q_{1}:=$ $\left(x_{1}, y_{1}\right), Q_{2}:=\left(x_{2}, y_{2}\right)$ and $Q_{3}:=\left(x_{3}, y_{3}\right)$ in $\mathbb{R}^{2}$ as shown in figure 1. Hence, the area of the triangle $Q_{1} Q_{2} Q_{3}$ is just the constant $A$ (10.8), that is, the Heron's formula. From figure 1 we obtain that

$$
\begin{aligned}
& x_{1}^{ \pm}\left(x_{2}, y_{2}, x_{3}, y_{3}, k_{1}, k_{2}, k_{3}\right)=x_{2}+k_{1} \cos \left(\alpha_{1} \pm \alpha_{2}\right)=x_{2}+k_{1}\left(\cos \alpha_{1} \cos \alpha_{2} \mp \sin \alpha_{1} \sin \alpha_{2}\right), \\
& y_{1}^{ \pm}\left(x_{2}, y_{2}, x_{3}, y_{3}, k_{1}, k_{2}, k_{3}\right)=y_{2}+k_{1} \sin \left(\alpha_{1} \pm \alpha_{2}\right)=y_{2}+k_{1}\left(\sin \alpha_{1} \cos \alpha_{2} \pm \cos \alpha_{1} \sin \alpha_{2}\right),
\end{aligned}
$$

and by introducing the triangle relations

$$
\sin \alpha_{2}=\frac{2 A}{k_{1} k_{3}}, \quad \cos \alpha_{2}=\frac{k_{1}^{2}+k_{3}^{2}-k_{2}^{2}}{2 k_{1} k_{3}}, \quad \sin \alpha_{1}=\frac{y_{3}-y_{2}}{k_{3}}, \quad \cos \alpha_{1}=\frac{x_{3}-x_{2}}{k_{3}},
$$

we directly recover the equations (10.7).

We remark that the result (10.7) can be applied to all LH systems of class $\mathrm{P}_{1}$. For instance, this can further be used so as to obtain a superposition rule for the Bernoulli differential equations (1.4) with $a_{1}^{R}(t)=0$, provided that a change of variables $(x, y) \rightarrow(r, \theta)$ mapping its VessiotGuldberg Lie algebra into $\mathrm{P}_{1}$ is given. Equivalently, we can repeat the above procedure for the LH algebra of Bernoulli differential equations without deriving a diffeomorphism. 


\subsection{Superposition rules for $\mathbf{L H}$ systems of classes $\mathbf{I}_{8} \simeq \mathfrak{i s o}(1,1)$ and $\mathbf{I}_{14 A}^{r=1} \simeq \mathfrak{h}_{2}$}

The class $\mathrm{I}_{14 A}^{r=1} \simeq \mathbb{R} \ltimes \mathbb{R}$ admits a LH algebra isomorphic to $\mathfrak{h}_{2}$ spanned by the functions given in table 1 with commutation relations (9.2). There does not exist a non-trivial Casimir for $\mathfrak{h}_{2}$, so precluding, in principle, the obtention of a superposition rule through the coalgebra approach. Nevertheless, this problem can be circumvented by considering an inclusion of $\mathrm{I}_{14 \mathrm{~A}}^{r=1}$ as a Lie subalgebra of a Lie algebra of another class admitting a LH algebra with a non-trivial Casimir.

There are several classes containing $\mathrm{I}_{14 A}^{r=1}$, e.g., $\mathrm{P}_{2}, \mathrm{I}_{4}, \mathrm{I}_{5}, \mathrm{I}_{8}, \mathrm{P}_{5}, \ldots$ (cf. see table 2 in [13]). Due to the simple form of the Casimir of the LH algebra $\overline{\mathfrak{i s o}}(1,1)$ of $\mathrm{I}_{8}$ and that superposition rules for $\mathfrak{s l}(2)-\mathrm{LH}$ systems on the plane were already studied in [19], we shall construct a new superposition rule for $\mathrm{LH}$ systems of class $\mathrm{I}_{8} \simeq \mathfrak{i s o}(1,1)$, obtaining as a byproduct the one corresponding to LH ones of class $\mathrm{I}_{14 A}^{r=1}$.

The LH algebra $\overline{\mathfrak{i s o}}(1,1)$ has commutation relations

$$
\left\{h_{1}, h_{2}\right\}_{\omega}=h_{0}, \quad\left\{h_{1}, h_{3}\right\}_{\omega}=-h_{1}, \quad\left\{h_{2}, h_{3}\right\}_{\omega}=h_{2}, \quad\left\{h_{0},\right\}_{\omega}=0,
$$

with respect to $\omega=\mathrm{d} x \wedge \mathrm{d} y$ in the basis $\left\{h_{1}, h_{2}, h_{3}, h_{0}\right\}$ given in table 1. We have that $m_{0}=2$ for a LH system $X$ with Vessiot-Gulgdberg Lie algebra $\mathrm{I}_{8}$. If $m=3$, then we obtain from table 2 three constants of motion for the diagonal prolongation $\widetilde{X}$ to $\left(\mathbb{R}^{2}\right)^{3}$ by applying (10.3):

$$
\begin{aligned}
& F^{(2)}=\left(x_{1}-x_{2}\right)\left(y_{1}-y_{2}\right)=k_{1}, \\
& F_{23}^{(2)}=\left(x_{1}-x_{3}\right)\left(y_{1}-y_{3}\right)=k_{2}, \\
& F_{13}^{(2)}=\left(x_{3}-x_{2}\right)\left(y_{3}-y_{2}\right)=k_{3} .
\end{aligned}
$$

Notice that $F^{(3)}=F_{12}^{(2)}+F_{13}^{(2)}+F_{23}^{(2)}$. In this case, $F^{(2)}=k_{1}, F_{23}^{(2)}=k_{2}$ can be understood as the equations on $\mathbb{R}^{2}$ of rectangular hyperbolas with centers $\left(x_{2}, y_{2}\right),\left(x_{3}, y_{3}\right)$. Clearly, $F^{(2)}$ and $F_{23}^{(2)}$ are functionally independent and allow us to express $\left(x_{1}, y_{1}\right)$ in terms of $\left(x_{2}, y_{2}, x_{3}, y_{3}\right)$ and $k_{1}, k_{2}$. The introduction of $k_{3}$ again simplifies the final result which reads

$$
\begin{aligned}
& x_{1}\left(x_{2}, y_{2}, x_{3}, y_{3}, k_{1}, k_{2}, k_{3}\right)=\frac{1}{2}\left(x_{2}+x_{3}\right)+\frac{k_{2}-k_{1} \pm B}{2\left(y_{2}-y_{3}\right)}, \\
& y_{1}\left(x_{2}, y_{2}, x_{3}, y_{3}, k_{1}, k_{2}, k_{3}\right)=\frac{1}{2}\left(y_{2}+y_{3}\right)+\frac{k_{2}-k_{1} \mp B}{2\left(x_{2}-x_{3}\right)},
\end{aligned}
$$

where

$$
B=\sqrt{k_{1}^{2}+k_{2}^{2}+k_{3}^{2}-2\left(k_{1} k_{2}+k_{1} k_{3}+k_{2} k_{3}\right)} .
$$

Consequently, if we recall that $k_{3}=k_{3}\left(x_{2}, y_{2}, x_{3}, y_{3}\right)$, we obtain that (10.9) leads to a superposition rule for LH systems with a Vessiot-Guldberg Lie algebra $\mathrm{I}_{14 A}^{r=1}$ provided

$$
k_{1}^{2}+k_{2}^{2}+k_{3}^{2} \geq 2\left(k_{1} k_{2}+k_{1} k_{3}+k_{2} k_{3}\right) .
$$

Let us now recover the superposition rule for a LH system of class $\mathrm{I}_{14 A}^{r=1}$ out of these results. It is immediate that up to a trivial change of variables $\mathrm{I}_{14 A}^{r=1}=\left\langle\partial_{u}, \mathrm{e}^{u} \partial_{v}\right\rangle$. Then, the change of variables

$$
y=\mathrm{e}^{u}, \quad x=v \mathrm{e}^{-u},
$$

maps the basis of $\mathrm{I}_{14 A}^{r=1}$ into vector fields $-X_{3}$ and $X_{1}$ of $\mathrm{I}_{8}$ given in table 1 . Hence, every LH system of class $\mathrm{I}_{14 A}^{r=1}$ can be considered as a $\mathrm{LH}$ system of class $\mathrm{I}_{8}$ and the above superposition rule for $\mathrm{I}_{8}$ also applies, up to a change of variables, to LH systems of class $\mathrm{I}_{14 A}^{r=1}$. It is worth noting that this can be applied to the specific systems of class $\mathrm{I}_{14 A}^{r=1}$ studied in section 9 . 


\subsection{Superposition rules for two-photon LH systems}

Finally, consider a LH system with a Vessiot-Guldberg Lie algebra $\mathrm{P}_{5}$. It can be proved that the prolongations of any basis of $\mathrm{P}_{5}$ become linearly independent at a generic point for $m_{0}=3$. So we fix $m=4$. The associated LH algebra $\mathfrak{h}_{6}$ with the basis given in table 1 fulfills the commutation relations (7.1). According to table 2, the constant of motion $F^{(2)}=0$. From $F^{(3)}$ written in table 2 and by using (10.3) we obtain four constants of motion given by

$$
\begin{aligned}
& F^{(3)}=\left(x_{1}\left(y_{2}-y_{3}\right)+x_{2}\left(y_{3}-y_{1}\right)+x_{3}\left(y_{1}-y_{2}\right)\right)^{2}=k_{1}^{2}, \\
& F_{34}^{(3)}=\left(x_{1}\left(y_{2}-y_{4}\right)+x_{2}\left(y_{4}-y_{1}\right)+x_{4}\left(y_{1}-y_{2}\right)\right)^{2}=k_{2}^{2}, \\
& F_{24}^{(3)}=\left(x_{1}\left(y_{3}-y_{4}\right)+x_{3}\left(y_{4}-y_{1}\right)+x_{4}\left(y_{1}-y_{3}\right)\right)^{2}=k_{3}^{2}, \\
& F_{14}^{(3)}=\left(x_{2}\left(y_{3}-y_{4}\right)+x_{3}\left(y_{4}-y_{2}\right)+x_{4}\left(y_{2}-y_{3}\right)\right)^{2}=k_{4}^{2},
\end{aligned}
$$

such that $F^{(4)}=F^{(3)}+F_{34}^{(3)}+F_{24}^{(3)}+F_{14}^{(3)}$. The constants of motion $F^{(3)}$ and $F^{(4)}$ are functionally independent functions, but also $F^{(3)}$ and $F_{34}^{(3)}$ which have a simpler form. Thus from the latter and by taking positive square roots we find $\left(x_{1}, y_{1}\right)$ written in terms of $\left(x_{2}, y_{2}, x_{3}, y_{3}, x_{4}, y_{4}\right)$ and the two constants $k_{1}$ and $k_{2}$. The result is rather simplified by introducing a third constant $k_{4}$ coming from the positive square root of $F_{14}^{(3)}$ (the remaining constant $k_{3}$ does not enter), yielding

$$
\begin{aligned}
& x_{1}\left(x_{2}, y_{2}, x_{3}, y_{3}, x_{4}, y_{4}, k_{1}, k_{2}, k_{4}\right)=\left(1+\frac{k_{2}-k_{1}}{k_{4}}\right) x_{2}-\frac{k_{2}}{k_{4}} x_{3}+\frac{k_{1}}{k_{4}} x_{4}, \\
& y_{1}\left(x_{2}, y_{2}, x_{3}, y_{3}, x_{4}, y_{4}, k_{1}, k_{2}, k_{4}\right)=\left(1+\frac{k_{2}-k_{1}}{k_{4}}\right) y_{2}-\frac{k_{2}}{k_{4}} y_{3}+\frac{k_{1}}{k_{4}} y_{4},
\end{aligned}
$$

which are well defined whenever $k_{4} \neq 0$. The above expression gives rise to a superposition rule for LH systems with a Vessiot-Guldberg Lie algebra $\mathrm{P}_{5}$ by considering $k_{4}$ as a function $k_{3}=k_{3}\left(x_{2}, y_{2}, x_{3}, y_{3}, x_{4}, y_{4}\right)$. Obviously, this result is also valid, up to an appropriate change of variables, to any other Lie system of class $\mathrm{P}_{5}$. In particular, this result can be applied to the two-photon LH systems described in section 7 , e.g., to $t$-dependent dissipative harmonic oscillators.

\section{Concluding remarks}

This work introduces the so-called Casimir tensor fields for certain finite-dimensional Lie algebras of vector fields. This allowed us to easily classify Lie algebras of vector fields on $\mathbb{R}^{2}$ isomorphic to $\mathfrak{s l}(2)$. In the future, we aim to extend our techniques to arbitrary finite-dimensional Lie algebras of vector fields. We also hope to devise methods to construct and to classify general LH systems in a systematic way.

We have presented new LH systems: different kinds of Bernoulli differential equations, Cayley-Klein Riccati equations, planar diffusion Riccati systems, etc. We have related them with some already known LH systems scattered in the literature. These results are summarized in table 3 where, according to the local classification of planar LH systems displayed in table 1 . we present the specific LH systems which are locally diffeomorphic within each class. For the sake of completeness, we also indicate LH systems that have not been studied here, but that can be found in [13, 18, 19, 28, 54]. As a result, table 3 details all LH systems on the plane with physical and mathematical applications appearing in the literature. Of course, the search of new applications of LH systems on the plane is still an open problem. 
Table 3: Specific LH systems on the plane according to their class given in table 1, All of these systems have $t$-dependent real coefficients except for $\mathrm{P}_{1}$. The systems marked with ' $*$ ' ( $\mathrm{I}_{14 A}^{r=2}$ and $\mathrm{I}_{14 B}^{r=2}$ ) have been studied in [13, while the one marked with ' $\dagger$ ' in $\mathrm{P}_{3}$ can be found in [19, 54].

\begin{tabular}{|c|c|c|}
\hline$\#$ & LH algebra & LH systems \\
\hline $\mathrm{P}_{1}$ & $\overline{\mathfrak{i s o}(2)}$ & Complex Bernoulli equation $\dot{z}=\mathrm{i} a(t) z+b(t) z^{n}$ for real $a(t)$ and complex $b(t)$ \\
\hline $\mathrm{P}_{2}$ & $\mathfrak{s l}(2)$ & $\begin{array}{l}\text { Complex Riccati equation } \\
\text { Milne-Pinney and Kummer-Schwarz equations with } c>0\end{array}$ \\
\hline $\mathrm{P}_{3}$ & $\mathfrak{s o}(3) \oplus \mathbb{R}, \mathfrak{s o}(3)$ & $\begin{array}{l}\text { Projective Schrödinger equations on } \mathbb{C P}^{1} \\
\text { Planar system with trigonometric nonlinearities }{ }^{\dagger}\end{array}$ \\
\hline $\mathrm{P}_{5}$ & $\mathfrak{h}_{6} \simeq \overline{\mathfrak{s l}(2) \ltimes \mathbb{R}^{2}}$ & $\begin{array}{l}\text { Dissipative/damped harmonic oscillators, particle under specific electric fields } \\
\text { Hamilton equations for quadratic Hamiltonians } \\
\text { Second-order Riccati equation in Hamiltonian form }\end{array}$ \\
\hline $\mathrm{I}_{4}$ & $\mathfrak{s l}(2)$ & $\begin{array}{l}\text { Split-complex Riccati equation } \\
\text { Coupled Riccati equations } \\
\text { Milne-Pinney and Kummer-Schwarz equations with } c<0 \\
\text { Planar diffusion Riccati system for } c_{0}=1\end{array}$ \\
\hline $\mathrm{I}_{5}$ & $\mathfrak{s l}(2)$ & $\begin{array}{l}\text { Dual-Study Riccati equation } \\
\text { Milne-Pinney and Kummer-Schwarz equations with } c=0 \\
\text { Harmonic oscillator } \\
\text { Planar diffusion Riccati system for } c_{0}=0\end{array}$ \\
\hline $\mathrm{I}_{14 A}^{r=1}$ & $\mathfrak{h}_{2} \simeq \mathbb{R} \ltimes \mathbb{R}$ & $\begin{array}{l}\text { Complex Bernoulli equation } \dot{z}=a_{1}(t) z+a_{2}(t) z^{n} \\
\text { Generalised Buchdahl equations } \\
\text { Lotka-Volterra systems }\end{array}$ \\
\hline $\mathrm{I}_{14 A}^{r=2}$ & $\mathbb{R} \ltimes \mathbb{R}^{2}$ & Quadratic polynomial systems $\dot{x}=b x+c(t) y+f(t) y^{2}, \dot{y}=y$ with $b \notin\{1,2\}^{*}$ \\
\hline $\mathrm{I}_{14 B}^{r=2}$ & $\overline{\mathbb{R} \ltimes \mathbb{R}^{2}}$ & $\begin{array}{l}\text { Quadratic polynomial systems } \dot{x}=b x+c(t) y+f(t) y^{2}, \dot{y}=y \text { with } b \in\{1,2\}^{*} \\
\text { A primitive model of viral infection* }\end{array}$ \\
\hline
\end{tabular}

Furthermore, we have derived $t$-independent constants of motion for most of the planar LH algebras by applying a coalgebra approach. This has been used to derive new superposition rules in an algebraic manner. In this respect, we remark that this procedure makes use of the non-deformed coproduct map (10.1). This fact naturally suggests us trying to extend such an approach to quantum (Poisson) algebras by considering deformed coproducts and deformed commutation rules. Thus the quantum deformation parameter $q$ would enter in the 'deformed' (generalized) LH systems in such a manner that the initial systems would be recovered under the non-deformed limit $q \rightarrow 1$.

Observe that the Casimir function $\mathrm{I}_{16}$ is the only element of table 2 that is not an element of $S\left(\overline{\mathfrak{h}_{2} \ltimes \mathbb{R}^{r+1}}\right)$. Therefore it cannot be straightforwardly employed through the theory of this work. Although our methods can be generalized by using the approach given in [47] for this type of Casimir elements, this approach does not provide any significant improvement for planar LH systems and it will left for studying problems where non-polynomial Casimir functions will be the rule rather than the exception.

Work on these lines is currently in progress. 


\section{Acknowledgments}

The research of A. Blasco and F.J. Herranz was partially supported by the Spanish Ministerio de Economía y Competitividad (MINECO) under grant MTM2013-43820-P and by Junta de Castilla y León under grant BU278U14. J. de Lucas and C. Sardón acknowledge funding from the Polish National Science Centre under the grant HARMONIA DEC-2012/04/M/ST1/00523. C. Sardón also acknowledges a fellowship provided by the University of Salamanca.

\section{References}

[1] Lie S and Scheffers G 1893 Vorlesungen über continuierliche Gruppen mit geometrischen und anderen Anwendungen (Leipzig: Teubner)

[2] Winternitz P 1983 Lie groups and solutions of nonlinear differential equations Nonlinear phenomena (Lectures Notes in Physics 189) (New York: Springer) pp 263-331

[3] Cariñena J F, Grabowski J and Marmo G 2000 Lie-Scheffers systems: a geometric approach (Naples: Bibliopolis)

[4] Cariñena J F and de Lucas J 2011 Lie systems: theory, generalisations, and applications, Dissertations Math. (Rozprawy Mat.) 479 1-162

[5] del Olmo M A, Rodríguez M and Winternitz P 1986 Integrability, chaos and nonlinear superposition formulas for differential matrix Riccati equations Quantum Chaos and Statistical Nuclear Physics (Lecture Notes in Physics 263) (New York: Springer) pp 372-378

[6] Abou-Kandil H, Freiling G, Ionescu V and Jank G 2003 Matrix Riccati equation in control and systems theory. (Systems \& control: Foundations \& Applications) (Berlin: Birkhäuser)

[7] Inselberg A 1972 Superpositions for nonlinear operators: I. Strong superpositions and linearizability J. Math. Anal. Appl. 40 494-508

[8] Carineña J F, Grabowski J and de Lucas J 2010 Lie families: theory and applications J. Phys. A: Math. Theor. 43305201

[9] Cariñena J F, Grabowski J and Marmo G 2007 Superposition rules, Lie theorem and partial differential equations Rep. Math. Phys. 60 237-258

[10] Marino A 2011 Topological methods, variational inequalities and elastic bounce trajectories Atti Accad. Naz. Lincei Cl. Sci. Fis. Mat. Natur. Rend. Lincei (9) Mat. Appl. 22 269-290

[11] Żołądek H 2000 The method of holomorphic foliations in planar periodic systems: the case of Riccati equations J. Differential Equations 165 143-173

[12] González-López A, Kamran N and Olver P J 1992 Lie algebras of vector fields in the real plane Proc. London Math. Soc. 64 339-368

[13] Ballesteros A, Blasco A, Herranz F J, de Lucas J and Sardón C 2015 Lie-Hamilton systems on the plane: Properties, classification and applications J. Differential Equations 258 2873-2907

[14] Lie S 1893 Theorie der Transformationsgruppen 3 (Leipzig: B G Teubner)

[15] Ackerman M and Hermann R 1975 Sophus Lie's 1880 transformation group paper (Brookline MA: Math. Sci. Press.)

[16] Vaisman I 1994 Lectures on the geometry of Poisson manifolds, Progress in Mathematics, 118, (Basel: Birkhäuser) 
[17] Marle C M 1997 The Schouten-Nijenhuis bracket and interior products J. Geom. Phys. 23 350-359

[18] Cariñena J F, de Lucas J and Sardón C 2013 Lie-Hamilton systems: theory and applications Int. J. Geom. Methods Mod. Phys. 101350047

[19] Ballesteros A, Cariñena J F, Herranz F J, de Lucas J and Sardón C 2013 From constants of motion to superposition rules for Lie-Hamilton systems J. Phys. A: Math. Theor. 46285203

[20] Estévez P G, Herranz F J, de Lucas J and Sardón C 2014 Lie symmetries for Lie systems: applications to systems of ODEs and PDEs Preprint arXiv:1404.2740

[21] Mariton M and Bertrand P 1985 A homotophy algorithm for solving coupled Riccati equations Optim. Control Appl. Meth. 6 351-357

[22] Leach P G L and Andriopoulos K 2008 The Ermakov equation: a commentary Appl. Anal. Discrete Math. 2 146-157

[23] Cariñena J F, Grabowski J and de Lucas J 2012 Superposition rules for higher-order systems and their applications J. Phys. A: Math. Theor. 45185202

[24] Suazo E, Suslov K S and Vega-Guzmán J M 2011 The Riccati differential equation and a diffusiontype equation New York J. Math. 17A 225-244

[25] Suazo E, Suslov K S and Vega-Guzmán J M 2014 The Riccati system and a diffusion-type equation Mathematics 2014 96-118

[26] Cariñena J F and Ramos A 2003 Applications of Lie systems in quantum mechanics and control theory Classical and Quantum Integrability Banach Center Publ. 59 143-162

[27] Um C I and Yeon K H 2002 Quantum Theory of the Harmonic Oscillator in Nonconservative Systems J. Korean Phys. Soc. 41 594-616.

[28] Cariñena J F, de Lucas J and Sardón C 2012 A new Lie system's approach to second-order Riccati equations Int. J. Geom. Methods Mod. Phys. 91260007

[29] Cariñena J F, Rañada M F and Santander M 2005 Lagrangian formalism for nonlinear second-order Riccati systems: one-dimensional integrability and two-dimensional superintegrability J. Math. Phys. 46062703

[30] Ballesteros A, Blasco A and Herranz F J 2009 N-dimensional integrability from two-photon coalgebra symmetry J. Phys. A: Math. Theor. 42265205.

[31] Buchdahl H A 1964 A relativistic fluid sphere resembling the Emden polytrope of index 5 Astrophys. J. $1401512-1516$

[32] Chandrasekar V K, Senthilvelan M and Lakshmanan M 2005 On the complete integrability and linearization of certain second-order nonlinear ordinary differential equations Proc. R. Soc. Lond. Ser. A Math. Phys. Eng. Sci. A 461 2451-2476

[33] Ciesliński J L and Nikiciuk T 2010 A direct approach to the construction of standard and nonstandard Lagrangians for dissipative-like dynamical systems with variable coefficients J. Phys. A: Math. Theor. 43175205

[34] Tsvetkov D P 1996 A periodic Lotka-Volterra system Serdica Math. J. 22 109-116

[35] Jin Z, Maoan H and Guihua L 2005 The persistence in a Lotka-Volterra competition systems with impulsive Chaos Solitons Fractals 24 1105-1117

[36] Zhang W M, Feng D H and Gilmore R 1990 Coherent states: theory and some applications Rev. Mod. Phys. 62 867-927 
[37] Ballesteros A, Herranz F J and Parashar P 2000 (1+1) Schrödinger Lie bialgebras and their PoissonLie groups, J. Phys. A: Math. Gen. 33 3445-3465.

[38] Cariñena J F and López C 1999 Group theoretical perturbative treatment of non linear Hamiltonians on the dual of a Lie algebra Rep. Math. Phys. 43 43-51.

[39] Varadarajan V S 1984 Lie Groups, Lie Algebras, and Their Representations (Graduate Texts in Mathematics vol 102) (New York: Springer)

[40] Ortega R 2012 The complex periodic problem for a Riccati equation Ann. Univ. Buchar. Math. Ser. $3219-226$

[41] Campos J 1997 Möbius transformations and periodic solutions of complex Riccati equations Bull. London Math. Soc. 29 205-215

[42] Farooq M U, Mahomed F M and Rashid M A 2010 Integration of systems of ODEs via nonlocal symmetry-like operators Math. Comput. Appl. 15 585-600

[43] Egorov A I 2007 Riccati equations Russian Academic Monographs 5 (Sofia-Moscow: Pensoft Publ.)

[44] Wilczyński P 2008 Planar nonautonomous polynomial equations: the Riccati equation J. Differential Equations 244 1304-1328

[45] Schuch D 2012 Complex Riccati equations as a link between different approaches for the description of dissipative and irreversible systems J. Phys.: Conf. Ser. 380012009

[46] Yaglom I M 1979 A simple non-Euclidean geometry and its physical basis (New York: Springer)

[47] Cariñena J F, Grabowski J, de Lucas J and Sardón C 2014 Dirac-Lie systems and Schwarzian equations J. Differential Equations 257 2303-2340

[48] Milne W E 1930 The numerical determination of characteristic numbers Phys. Rev. 35 863-867

[49] Pinney E 1950 The nonlinear differential equation $y^{\prime \prime}+p(x) y+c y^{-3}=0$ Proc. Amer. Math. Soc. 1681

[50] Friš J, Mandrosov V, Smorodinsky Y A, Uhlî́ M and Winternitz P 1965 On higher symmetries in quantum mechanics Phys. Lett. 16 354-356

[51] Ballesteros A, Herranz F J and Musso F 2013 The anisotropic oscillator on the 2D sphere and the hyperbolic plane Nonlinearity $\mathbf{2 6} 971-990$

[52] Muriel C and Romero J L $2014 \lambda$-symmetries of some chains of ordinary differential equations Nonlinear Analysis: Real World Applications 16 191-201

[53] Chari V and Pressley A 1994 A guide to quantum groups (Cambridge: Cambridge University Press)

[54] Angelo R M, Duzzioni E I, and Ribeiro A D 2012 Integrability in t-dependent systems with one degree of freedom J. Phys. A: Math. Theor. 45055101 